\title{
Synthesis and Biological Evaluation of Novel
}

\section{Naphthocarbazoles as Potential Anticancer Agents}

Sylvain Routier, ${ }^{a},{ }^{*}$ Paul Peixoto, ${ }^{a}$ Jean-Yves Mérour, ${ }^{a}$ Gérard Coudert, ${ }^{a}$ Nathalie Dias, ${ }^{b}$ Christian Bailly, ${ }^{b}$ Alain Pierré, ${ }^{c}$ Stéphane Léonce, ${ }^{c}$ Daniel-Henry Caignard. ${ }^{c}$

${ }^{\text {a } I n s t i t u t ~ d e ~ C h i m i e ~ O r g a n i q u e ~ e t ~ A n a l y t i q u e, ~ U M R ~ C N R S ~ 6005, ~ U n i v e r s i t e ́ ~ d ' o r l e ́ a n s, ~ R u e ~ d e ~ C h a r t r e s, ~}$

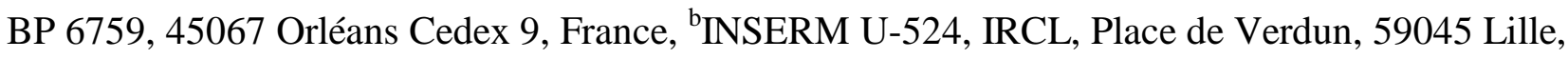
France, ${ }^{\mathrm{I}}$ Institut de Recherches Servier, Division Recherche Cancérologie, 125 chemin de Ronde, 78290 Croissy sur Seine, France

Table of contents :

Spectroscopic data.

Elemental analysis Table of related compounds

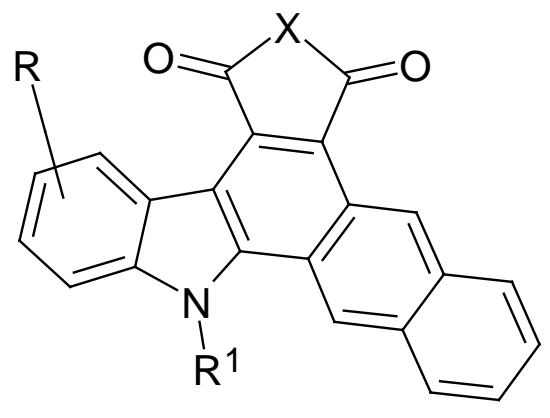

corresponding author : sylvain.routier@univ-orleans.fr 
3-(1-Benzenesulfonyl-1H-indol-3-yl)-4-bromo-1-methyl-pyrrole-2,5-dione (3). Mp $128{ }^{\circ} \mathrm{C} ; \mathrm{Rf}$ (petroleum ether/EtOAc 3/7) : 0.84 ; IR (KBr) v 3076, 2938, 1704, 1377, 1175, 984, $754 ;{ }^{1} \mathrm{H}$ NMR $\left(\mathrm{CDCl}_{3}\right) 3.17(\mathrm{~s}, 3 \mathrm{H}), 7.27-7.52(\mathrm{~m}, 5 \mathrm{H}), 7.80(\mathrm{~d}, 1 \mathrm{H}, J=9.2), 7.95(\mathrm{~m}, 3 \mathrm{H}), 8.26(\mathrm{~s}, 1 \mathrm{H}) ;{ }^{13} \mathrm{C} \mathrm{NMR}$ $\left(\mathrm{CDCl}_{3}\right) 27.6\left(\mathrm{CH}_{3}\right), 112.8(\mathrm{Cq}), 116.1(\mathrm{CH}), 124.1(\mathrm{Cq}), 125.5(\mathrm{CH}), 126.5(\mathrm{CH}), 128.5(\mathrm{CH}), 129.7$ $(2 \mathrm{CH}), 129.8(\mathrm{Cq}), 132.1(\mathrm{CH}), 132.2(2 \mathrm{CH}), 137.1(\mathrm{CH}), 137.4(\mathrm{Cq}), 138.4(\mathrm{Cq}), 140.1(\mathrm{Cq}), 168.2$ (CO) , $171.0(\mathrm{CO})$; MS (IS) : 445, $447(\mathrm{M}+1)^{+}$.

(3-Methoxy-naphthalen-2-yl)-trimethyl-stannane (4). Mp $64{ }^{\circ} \mathrm{C}$; Rf (petroleum ether) : 0.74 ; IR $(\mathrm{KBr}) \vee 3062,2988,2906,2840,1632,1594,1455,1204,1049,784,754 ;{ }^{1} \mathrm{H} \mathrm{NMR}\left(\mathrm{CDCl}_{3}\right) 0.32(\mathrm{t}$, 9H, $J=46.5 \mathrm{~Hz}), 3.94(\mathrm{~s}, 3 \mathrm{H}), 7.02(\mathrm{~s}, 1 \mathrm{H}), 7.32(\mathrm{t}, 1 \mathrm{H}, J=6.7), 7.42(\mathrm{t}, 1 \mathrm{H}, J=6.7), 7.7(\mathrm{~m}, 2 \mathrm{H}), 7.83$ (s, $1 \mathrm{H}) ;{ }^{13} \mathrm{C} \mathrm{NMR}\left(\mathrm{CDCl}_{3}\right)-3.82\left(3 \mathrm{CH}_{3}\right), 57.5\left(\mathrm{CH}_{3}\right), 105.4(\mathrm{CH}), 125.5(\mathrm{CH}), 128.5(\mathrm{CH}), 128.9(\mathrm{CH})$, $130.7(\mathrm{CH}), 132.4(\mathrm{Cq}), 135.8(\mathrm{Cq}), 137.6(\mathrm{Cq}), 139.4(\mathrm{CH}), 164.3(\mathrm{Cq}), \mathrm{MS}(\mathrm{IS}): 322(\mathrm{M}+1)^{+}$.

(3-Methoxy-naphthalen-2-yl)-boronic acid (5) Mp $148{ }^{\circ} \mathrm{C}$; Rf (petroleum ether/EtOAc 6/4) : 0.54 ; IR (KBr) v 3372, 2922, 1640, 1454, 1344, 1270, 1218, $762 ;{ }^{1} \mathrm{H}$ NMR (DMSO- $\left.d_{6}+\mathrm{D}_{2} \mathrm{O}\right) 3.90(\mathrm{~s}, 3 \mathrm{H})$, $7.29(\mathrm{t}, 1 \mathrm{H}, J=8.0), 7.42(\mathrm{t}, 1 \mathrm{H}, J=8.0), 7.74-7.78(\mathrm{~m}, 2 \mathrm{H}), 7.90(\mathrm{~s}, 1 \mathrm{H}), 8.09(\mathrm{~s}, 1 \mathrm{H}) ;{ }^{13} \mathrm{C} \mathrm{NMR}$ (DMSO- $\left.d_{6}+\mathrm{D}_{2} \mathrm{O}\right) 55.2\left(\mathrm{CH}_{3}\right), 104.5(\mathrm{CH}), 123.7(\mathrm{CH}), 126.2(\mathrm{CH}), 127.4(\mathrm{CH}), 128.3(\mathrm{CH}+\mathrm{Cq})$, $128.5(\mathrm{Cq}), 135.8(\mathrm{Cq}), 138.6(\mathrm{CH}), 160.9(\mathrm{Cq})$; MS (IS) : $203(\mathrm{M}+1)^{+}$.

3-(1H-Indol-3-yl)-4-(3-methoxy-naphthalen-2-yl)-1-methyl-pyrrole-2,5-dione (8). Mp $231{ }^{\circ} \mathrm{C}$; Rf (petroleum ether/EtOAc 5/5) : 0.44 ; IR (KBr) v 3370, 3052, 2946, 1706, 1630, 1524, 1432, 1204 , 1070, $746 ;{ }^{1} \mathrm{H}$ NMR $\left(\mathrm{CDCl}_{3}\right) 3.19$ (s, 3H), 3.37 (s, 3H), 6.59 (m, 2H), $7.03(\mathrm{~m}, 2 \mathrm{H}), 7.25-7.33(\mathrm{~m}, 2 \mathrm{H})$, $7.45(\mathrm{t}, 1 \mathrm{H}, J=8.0), 7.70(\mathrm{~m}, 2 \mathrm{H}), 7.91(\mathrm{~s}, 1 \mathrm{H}), 7.98(\mathrm{~d}, 1 \mathrm{H}, J=2.7), 8.69\left(\mathrm{~s}, 1 \mathrm{H}\right.$, exchangeable $\left.\mathrm{D}_{2} \mathrm{O}\right)$ $;^{13} \mathrm{C}$ NMR $\left(\mathrm{CDCl}_{3}\right) 24.3\left(\mathrm{CH}_{3}\right), 55.0\left(\mathrm{CH}_{3}\right), 106.0(\mathrm{CH}), 108.1(\mathrm{Cq}), 114.1(\mathrm{CH}), 120.7(\mathrm{CH}), 121.3$ $(\mathrm{CH}), 121.8(\mathrm{Cq}), 122.7(\mathrm{CH}), 123.9(\mathrm{CH}), 125.2(\mathrm{Cq}), 126.5(\mathrm{CH}), 127.1(\mathrm{CH}), 127.3(\mathrm{Cq}), 128.2$ 
$(\mathrm{CH}), 129.2(\mathrm{CH}), 130.3(\mathrm{Cq}), 131.7(\mathrm{CH}), 134.1(\mathrm{Cq}), 135.0(\mathrm{Cq}), 136.0(\mathrm{Cq}), 155.9(\mathrm{Cq}), 171.7(\mathrm{CO})$ , $171.9(\mathrm{CO})$; MS (IS) : $383(\mathrm{M}+1)^{+}$.

\section{3-(1-Benzenesulfonyl-1H-indol-3-yl)-4-(3-methoxy-naphthalen-2-yl)-1-methyl-pyrrole-2,5-dione}

(10). Obtained from 16 as described for compound 3 in $96 \%$ yield. Mp $204{ }^{\circ} \mathrm{C}$; Rf. (petroleum ether/EtOAc 6/4) : 0.84 ; IR (KBr) v 3152, 3070, 2940, 1722, 1450, 1396, 1184, 988, $754 ;{ }^{1} \mathrm{H}$ NMR $\left(\mathrm{CDCl}_{3}\right) 2.79(\mathrm{~s}, 3 \mathrm{H}), 3.20(\mathrm{~s}, 3 \mathrm{H}), 6.58(\mathrm{~d}, 1 \mathrm{H}, J=8.0), 6.74,(\mathrm{t}, 1 \mathrm{H}, J=7.2), 6.89(\mathrm{~s}, 1 \mathrm{H}), 7.17(\mathrm{t}, 1 \mathrm{H}$, $J=7.2), 7.36-7.53(\mathrm{~m}, 5 \mathrm{H}), 7.65(\mathrm{~d}, 1 \mathrm{H}, J=8.0), 7.77(\mathrm{~d}, 1 \mathrm{H}, J=7.2), 7.88(\mathrm{dd}, 2 \mathrm{H}, J=7.0, J=1.2)$, $7.99(\mathrm{~m}, 2 \mathrm{H}), 8.16(\mathrm{~s}, 1 \mathrm{H}){ }^{13} \mathrm{C}$ NMR $\left(\mathrm{CDCl}_{3}\right) 24.8\left(\mathrm{CH}_{3}\right), 54.7\left(\mathrm{CH}_{3}\right), 106.3(\mathrm{CH}), 113.7(\mathrm{CH}), 114.0$ $(\mathrm{Cq}), 120.7(\mathrm{CH}), 121.7(\mathrm{Cq}), 123.8(\mathrm{CH}), 124.7(\mathrm{CH}), 125.3(\mathrm{CH}), 126.8(\mathrm{CH}), 127.4(\mathrm{CH}), 128.0$ $(2 \mathrm{CH}), 128.4(\mathrm{CH}), 128.6(\mathrm{Cq}), 129.0(2 \mathrm{Cq}), 129.8(2 \mathrm{CH}+\mathrm{Cq}), 132.2(\mathrm{Cq}), 132.4(\mathrm{CH}), 134.5(\mathrm{CH})$, $135.0(\mathrm{Cq}), 135.5(\mathrm{Cq}), 138.1(\mathrm{Cq}), 155.7(\mathrm{CH}), 171.0(\mathrm{CO}), 171.3(\mathrm{CO})$; MS (IS) : $523(\mathrm{M}+1)^{+}$.

3-(3-Hydroxy-naphthalen-2-yl)-4-(1H-indol-3-yl)-1-methyl-pyrrole-2,5-dione (11). Mp $165{ }^{\circ} \mathrm{C}$; Rf (petroleum ether/EtOAc 5/5) : 0.34 ; IR (KBr) v 3398, 3162, 3029, 1705, 1632, 1448, 1231, 990, $751 ;{ }^{1} \mathrm{H}$ NMR $\left(\mathrm{CDCl}_{3}\right) 3.06(\mathrm{~s}, 3 \mathrm{H}), 6.50(\mathrm{~m}, 2 \mathrm{H}), 6.93(\mathrm{t}, 1 \mathrm{H}, J=7.5), 7.17(\mathrm{~s}, 1 \mathrm{H}), 7.24(\mathrm{t}, 1 \mathrm{H}, J=$ 7.5), $7.35(\mathrm{~m}, 2 \mathrm{H}), 7.73(\mathrm{~m}, 3 \mathrm{H}), 7.97(\mathrm{~s}, 1 \mathrm{H}), 9.93\left(\mathrm{~s}, 1 \mathrm{H}\right.$, exchangeable $\left.\mathrm{D}_{2} \mathrm{O}\right), 11.89(\mathrm{~s}, 1 \mathrm{H}$, exchangeable $\left.\mathrm{D}_{2} \mathrm{O}\right) ;{ }^{13} \mathrm{C}$ NMR (DMSO- $\left.d_{6}\right) 24.89\left(\mathrm{CH}_{3}\right), 106.5(\mathrm{Cq}), 109.9(\mathrm{CH}), 112.8(\mathrm{CH}), 120.7$ $(\mathrm{CH}), 121.4(\mathrm{CH}), 122.5(\mathrm{Cq}), 122.8(\mathrm{Cq}), 123.9(\mathrm{Cq}), 125.9(\mathrm{CH}), 126.6(\mathrm{Cq}), 127.5(\mathrm{CH}), 127.8(\mathrm{Cq})$, $128.7(\mathrm{CH}), 131.5(\mathrm{CH}), 131.6(\mathrm{CH}), 135.3(\mathrm{CH}), 135.4(\mathrm{Cq}), 137.2(\mathrm{CH}), 147.5(\mathrm{Cq}), 154.7(\mathrm{CH})$, $171.9(\mathrm{CO}), 172.2(\mathrm{CO})$; MS (IS) : $369(\mathrm{M}+1)^{+}$.

\section{3-(1-Benzenesulfonyl-1H-indol-3-yl)-4-(3-hydroxy-naphthalen-2-yl)-1-methyl-pyrrole-2,5-dione}

(12). Mp $192{ }^{\circ} \mathrm{C} ; \operatorname{Rf}($ petroleum ether/EtOAc 4/6) : 0.72 ; $\mathbb{R}(\mathrm{KBr}) \vee 3388,3156,3050,1932,1703$, 1447, 1385, 1222, 1176, 993 ; chromatography (petroleum ether/EtOAc 5 /5) to afford compound 12 as a yellow solid (195 mg, $98 \%) .{ }^{1} \mathrm{H}$ NMR $\left(\mathrm{CDCl}_{3}\right) 3.24$ (s, 3H), 6.45 (d, $\left.1 \mathrm{H}, J=8.2\right), 6.65(\mathrm{t}, 1 \mathrm{H}, J=$ 7.1), 7.09-7.21 (m, 2H), 7.29 (s, 1H, exchangeable $\left.\mathrm{D}_{2} \mathrm{O}\right)$, 7.27-7.42 (m, 5H), 7.56 (s, 1H), 7.58-7.62 (m, 
2H), $7.86(\mathrm{~m}, 3 \mathrm{H}), 8.38(\mathrm{~s}, 1 \mathrm{H}) ;{ }^{13} \mathrm{C} \mathrm{NMR}\left(\mathrm{CDCl}_{3}\right) 24.9\left(\mathrm{CH}_{3}\right), 112.2(\mathrm{CH}), 112.7(\mathrm{Cq}), 113.7(\mathrm{CH})$, $120.0(\mathrm{Cq}), 122.3(\mathrm{CH}), 123.9(\mathrm{CH}), 124.4(\mathrm{CH}), 125.5(\mathrm{CH}), 126.5(\mathrm{CH}), 127.3(2 \mathrm{CH}), 127.5(\mathrm{Cq})$, $128.0(\mathrm{CH}), 128.5(\mathrm{CH}+\mathrm{Cq}), 129.8(\mathrm{CH}), 130.5(2 \mathrm{CH}), 132.5(\mathrm{Cq}), 132.6(\mathrm{Cq}), 133.8(\mathrm{CH}), 134.6$ $(\mathrm{Cq}), 135.2(\mathrm{Cq}), 135.8(\mathrm{Cq}), 137.9(\mathrm{Cq}), 152.11(\mathrm{CH}), 170.7(\mathrm{CO}), 173.0(\mathrm{CO})$; MS (IS) : 509 $(\mathrm{M}+1)^{+}$.

\section{Trifluoromethanesulfonic acid 3-[4-(1H-indol-3-yl)-1-methyl-2,5-dioxo-2,5-dihydro-1H-pyrrol-}

3-yl]-naphthalen-2-yl ester (13). Mp $155{ }^{\circ} \mathrm{C}$; Rf (petroleum ether/ EtOAc $\left.4 / 6, \mathrm{NEt}_{3} 10 \%\right) 0.86$; IR $(\mathrm{KBr}) \vee 3398,3161,3029,1703,1630,1493,1448,1385,1231,1091,751 ;{ }^{1} \mathrm{H} \mathrm{NMR}\left(\mathrm{CDCl}_{3}\right) 3.24$ (s, 3H), $6.38(\mathrm{~d}, 1 \mathrm{H}, J=8.1), 6.55(\mathrm{t}, 1 \mathrm{H}, J=7.9), 7.01(\mathrm{t}, 1 \mathrm{H}, J=8.1), 7.28(\mathrm{~d}, 1 \mathrm{H}, J=8.1), 7.56-7.66$ (m, 3H), $7.82(\mathrm{t}, 1 \mathrm{H}, J=5.0), 7.91(\mathrm{t}, 1 \mathrm{H}, J=4.3), 8.09(\mathrm{~d}, 1 \mathrm{H}, J=2.9), 8.18(\mathrm{~s}, 1 \mathrm{H}), 9.08(\mathrm{~s}, 1 \mathrm{H}$, exchangeable $\left.\mathrm{D}_{2} \mathrm{O}\right) ;{ }^{13} \mathrm{C}$ NMR $\left.\left(\mathrm{CDCl}_{3}\right) 25.0 \mathrm{CH}_{3}\right), 106.0(\mathrm{Cq}), 113.3(\mathrm{CH}), 114.2(\mathrm{Cq}), 120.8(\mathrm{CH})$, $120.9(\mathrm{CH}), 121.1(\mathrm{CH}), 122.6(\mathrm{Cq}), 123.2(\mathrm{Cq}), 123.5(\mathrm{CH}), 125.4(\mathrm{CH}), 128.9(2 \mathrm{CH}), 129.1(\mathrm{CH})$, $129.3(\mathrm{Cq}), 132.4(\mathrm{Cq}), 133.0(\mathrm{CH}), 133.6(\mathrm{CH}), 133.8(\mathrm{Cq}), 136.6(\mathrm{Cq}), 137.5(\mathrm{Cq}), 145.6(\mathrm{Cq}), 171.1$ (CO), $171.3(\mathrm{CO})$; MS (IS) : $501(\mathrm{M}+1)^{+}$.

\section{Trifluoro-methanesulfonic acid 3-[4-(1-benzenesulfonyl-1H-indol-3-yl)-1-methyl-2,5-dioxo-2,5-} dihydro-1 H-pyrrol-3-yl]-naphthalen-2-yl ester (14). $\mathrm{Mp} 96{ }^{\circ} \mathrm{C}$; $\mathrm{Rf}$ (petroleum ether/EtOAc 5/5, $\mathrm{NEt}_{3}$ $10 \%): 0.88$; IR $(\mathrm{KBr}) \vee 3142,3028,2993,2905,1769,1713,1602,1446,1385,1139,1039,891$; \%). ${ }^{1} \mathrm{H}$ NMR $\left(\mathrm{CDCl}_{3}\right) 3.25(\mathrm{~s}, 3 \mathrm{H}), 6.44(\mathrm{~d}, 1 \mathrm{H}, J=8.2), 6.66(\mathrm{t}, 1 \mathrm{H}, J=7.5), 7.13(\mathrm{t}, 1 \mathrm{H}, J=7.5), 7.25-$ $7.64(\mathrm{~m}, 6 \mathrm{H}), 7.78(\mathrm{~m}, 1 \mathrm{H}), 7.92(\mathrm{~m}, 4 \mathrm{H}), 8.18(\mathrm{~s}, 1 \mathrm{H}), 7.35(\mathrm{~s}, 1 \mathrm{H}) ;{ }^{13} \mathrm{C} \mathrm{NMR}\left(\mathrm{CDCl}_{3}\right) 24.6\left(\mathrm{CH}_{3}\right)$, $111.1(\mathrm{Cq}), 113.4(\mathrm{CH}), 117.3(\mathrm{Cq}), 120.0(\mathrm{Cq}), 120.9(\mathrm{CH}), 121.6(\mathrm{CH}), 123.4(\mathrm{CH}), 125.0(\mathrm{CH})$, $122.0(2 \mathrm{CH}), 127.3(\mathrm{Cq}), 127.7(\mathrm{CH}), 127.9(\mathrm{CH}), 128.6(\mathrm{CH}), 128.7(\mathrm{CH}+\mathrm{Cq}), 129.3(2 \mathrm{CH}), 130.6$ $(\mathrm{CH}), 131.7(\mathrm{Cq}), 133.0(\mathrm{CH}), 133.2(\mathrm{Cq}), 133.4(\mathrm{Cq}), 134.2(\mathrm{CH}), 134.6(\mathrm{Cq}), 137.4(\mathrm{Cq}), 144.6(\mathrm{Cq})$, $169.6(\mathrm{CO}), 169.8(\mathrm{CO})$; MS (IS) : $641(\mathrm{M}+1)^{+}$. 
6-Methylnaphtho[2,3-a]pyrrolo[3,4-c]carbazole-5,7(6H,14H)-dione (15). Mp 250 $\mathrm{C}$ dec. ;

Rf (petroleum ether /EtOAc 2/6) : 0.24 ; IR (KBr) v 1381, 1443, 1680, 1748, 2925, 3052, 3552 (NH) ;

${ }^{1} \mathrm{H}$ NMR (DMSO-d $) 3.00(\mathrm{~s}, 3 \mathrm{H}), 7.34(\mathrm{t}, 1 \mathrm{H}, J=10.0 \mathrm{~Hz}), 7.62(\mathrm{t}, 1 \mathrm{H}, J=7.5 \mathrm{~Hz}), 7.57-7.68(\mathrm{~m}$, 3H), $8.04(\mathrm{~d}, 1 \mathrm{H}, J=7.5 \mathrm{~Hz}), 8.13(\mathrm{~d}, 1 \mathrm{H}, J=10.0 \mathrm{~Hz}), 8.78(\mathrm{~d}, 1 \mathrm{H}, J=10.0 \mathrm{~Hz}), 9.08(\mathrm{~s}, 1 \mathrm{H}), 9.36(\mathrm{~s}$, 1H), 13.01 (br s, 1H, NH). ${ }^{13} \mathrm{C}$ NMR (DMSO-d $) 25.7\left(\mathrm{CH}_{3}\right), 110.2(\mathrm{Cq}), 112.6(\mathrm{CH}), 118.8(\mathrm{Cq}), 121.7$ $(\mathrm{CH}), 121.8(\mathrm{Cq}), 112.2(\mathrm{CH}), 122.5(\mathrm{Cq}), 124.3(\mathrm{CH}), 124.9(\mathrm{CH}), 126.5(\mathrm{CH}), 127.5(\mathrm{CH}), 127.8$ $(\mathrm{CH}), 128.9(\mathrm{CH}), 129.5(2 \mathrm{Cq}), 132.1(\mathrm{CH}), 132.4(\mathrm{Cq}), 132.6(\mathrm{Cq}), 140.0(\mathrm{Cq}), 140.8(\mathrm{Cq}), 169.9$ $(\mathrm{Cq}), 170.8(\mathrm{Cq}) . \mathrm{MS}(\mathrm{IS}) 351(\mathrm{M}+\mathrm{H})^{+}$.

\section{6-Methyl-14-benzenesulfonyl-naphtho[2,3-a]pyrrolo[3,4-c $]$ carbazole-5,7(6H,14H)-dione (16). Mp} $250^{\circ} \mathrm{C}$ dec. ; Rf (petroleum ether/EtOAc 7/3) : 0.34 ; IR (KBr) v 2938, 2852, 1716, 1452, 1394, 1182 , 1084, 826, 774, $674 ;{ }^{1} \mathrm{H}$ NMR (DMSO-d $) 3.10(\mathrm{~s}, 3 \mathrm{H}), 6.90(\mathrm{~d}, 2 \mathrm{H}, J=8.0 \mathrm{~Hz}), 7.62(\mathrm{t}, 2 \mathrm{H}, J=8.0$ Hz), 7.37-7.73 (m, 5H), $8.22(\mathrm{~m}, 3 \mathrm{H}), 8.13(\mathrm{~d}, 1 \mathrm{H}, J=8.0 \mathrm{~Hz}), 9.42(\mathrm{~s}, 1 \mathrm{H}), 9.55(\mathrm{~s}, 1 \mathrm{H})$. Compound of low solubility; degradation in DMSO at $80{ }^{\circ} \mathrm{C}$, no ${ }^{13} \mathrm{C}$ available. MS (IS) $491(\mathrm{M}+\mathrm{H})^{+}$.

\section{3-(1-Benzenesulfonyl-5-benzyloxy-1H-indol-3-yl)-4-bromo-1-methyl-pyrrole-2,5-dione (17). Mp} $162{ }^{\circ} \mathrm{C} ; \operatorname{Rf}($ petroleum ether/EtOAc 6/4) : 0.24 ; IR (KBr) v 3138, 3204, 2980, 1786, 1716, 1465, 1004 ;. ${ }^{1} \mathrm{H}$ NMR $\left(\mathrm{CDCl}_{3}\right) 3.13(\mathrm{~s}, 3 \mathrm{H}), 5.08(\mathrm{~s}, 2 \mathrm{H}), 7.14(\mathrm{dd}, 1 \mathrm{H}, J=2.2, J=9.0), 7.38(\mathrm{~m}, 6 \mathrm{H}), 7.63(\mathrm{~m}$, 2H), $7.74(\mathrm{~m}, 1 \mathrm{H}), 7.90(\mathrm{~d}, 1 \mathrm{H}, J=9.0), 8.06(\mathrm{~d}, 2 \mathrm{H}, J=7.1), 8.18(\mathrm{~s}, 1 \mathrm{H}) ;{ }^{13} \mathrm{C} \mathrm{NMR}\left(\mathrm{CDCl}_{3}\right) 25.4$ $\left(\mathrm{CH}_{3}\right), 70.8\left(\mathrm{CH}_{2}\right), 106.9(\mathrm{CH}), 110.6(\mathrm{Cq}), 114.8(\mathrm{CH}), 116.2(\mathrm{CH}), 121.4(\mathrm{Cq}), 126.3(\mathrm{Cq}), 127.4$ (2CH), $127.8(2 \mathrm{CH}), 128.4(\mathrm{CH}), 128.6(\mathrm{Cq}), 129.0(2 \mathrm{CH}), 129.9(2 \mathrm{CH}), 130.6(\mathrm{CH}), 134.8(\mathrm{CH})$, $136.2(\mathrm{Cq}), 137.1(\mathrm{Cq}), 137.9(\mathrm{Cq}), 156.1(\mathrm{Cq}), 166.2(\mathrm{CO}), 168.8(\mathrm{CO})$; MS (IS) : 551, $553(\mathrm{M}+1)^{+}$.

\section{3-(1-Benzenesulfonyl-6-benzyloxy-1H-indol-3-yl)-4-bromo-1-methyl-pyrrole-2,5-dione (18). Mp} $112{ }^{\circ} \mathrm{C}$; Rf (petroleum ether/EtOAc 6/4) : 0.55 ; IR (KBr) v 3064, 2944, 1775, 1685, 1613, 1447, 1383, 1170, 995, $725 ;{ }^{1} \mathrm{H}$ NMR $\left(\mathrm{CDCl}_{3}\right) 3.15$ (s, 3H), $5.21(\mathrm{~s}, 2 \mathrm{H}), 7.02(\mathrm{dd}, 1 \mathrm{H}, J=8.9, J=2.4), 7.35-7.62$ $(\mathrm{m}, 9 \mathrm{H}), 7.71(\mathrm{~d}, 1 \mathrm{H}, J=8.9), 7.76(\mathrm{~d}, 2 \mathrm{H}, J=7.9), 8.26(\mathrm{~s}, 1 \mathrm{H}) ;{ }^{13} \mathrm{C} \mathrm{NMR}\left(\mathrm{CDCl}_{3}\right) 25.3\left(\mathrm{CH}_{3}\right), 70.8$ 
$\left(\mathrm{CH}_{2}\right), 99.1(\mathrm{CH}), 110.9(\mathrm{Cq}), 114.4(\mathrm{CH}), 121.4(\mathrm{CH}), 121.5(\mathrm{Cq}), 124.0(\mathrm{CH}), 127.3(2 \mathrm{CH}), 127.7$

(2CH), $128.4(\mathrm{CH}), 129.0(\mathrm{Cq}), 129.1(2 \mathrm{CH}), 129.9(2 \mathrm{CH}), 134.7(\mathrm{CH}), 136.2(\mathrm{Cq}), 136.3(\mathrm{Cq}), 137.2$ (Cq), $137.7(\mathrm{Cq}), 157.8(\mathrm{Cq}), 166.1(\mathrm{CO}), 168.8(\mathrm{CO})$; MS (IS) : 551, $553(\mathrm{M}+1)^{+}$.

(3-Hydroxy-naphthalen-2-yl)-boronic acid (20). $\mathrm{Mp} 123{ }^{\circ} \mathrm{C} ; \operatorname{Rf}(\mathrm{EtOAc}): 0.45 ; \mathrm{IR}(\mathrm{KBr}) \vee 3378$, 3048, 1636, 1566, 1496, 862, $726 ;{ }^{1} \mathrm{H}$ NMR (DMSO- $\left.d_{6}+\mathrm{D}_{2} \mathrm{O}\right) 7.10(\mathrm{~s}, 1 \mathrm{H}), 7.23(\mathrm{t}, 1 \mathrm{H}, J=7.2), 7.38$ $(\mathrm{t}, 1 \mathrm{H}, J=7.9), 7.61\left((\mathrm{~d}, 1 \mathrm{H}, J=7.2), 7.76(\mathrm{~d}, 1 \mathrm{H}, J=8.0), 8.17(\mathrm{~s}, 1 \mathrm{H}) ;{ }^{13} \mathrm{C}\right.$ NMR $\left(\mathrm{CDCl}_{3}\right) 109.5$ $(\mathrm{CH}), 124.5(\mathrm{CH}), 126.9(\mathrm{CH}), 128.7(\mathrm{CH}), 128.8(\mathrm{Cq}), 129.4(\mathrm{CH}), 137.0(2 \mathrm{Cq}), 138.4(\mathrm{CH}), 159.8$ (Cq) ; MS (IS) : $189(\mathrm{M}+1)^{+}$.

\section{3-(1-Benzenesulfonyl-5-benzyloxy-1H-indol-3-yl)-4-(3-hydroxy-naphthalen-2-yl)-1-methyl-}

pyrrole-2,5-dione (21). $\mathrm{Mp} 210{ }^{\circ} \mathrm{C}$; Rf (petroleum ether: EtOAc 6:4) : 0.26 ; IR (KBr) v 3417, 3160, 3032, 2918, 1752, 1694, 1452, 1154, 990, $734 ;{ }^{1} \mathrm{H}$ NMR $\left(\mathrm{CDCl}_{3}\right) 3.24$ (s, 3H), 5.88 (s, 2H), 6.80 (d, $1 \mathrm{H}, J=8.7), 6.97(\mathrm{~m}, 2 \mathrm{H}), 7.16-7.63(\mathrm{~m}, 14 \mathrm{H}), 7.80(\mathrm{~d}, 1 \mathrm{H}, J=9.0), 7.95(\mathrm{~d}, 2 \mathrm{H}, J=7.5), 8.40(\mathrm{~s}, 1 \mathrm{H})$ ; ${ }^{13} \mathrm{C}$ NMR $\left(\mathrm{CDCl}_{3}\right) 24.8\left(\mathrm{CH}_{3}\right), 69.7\left(\mathrm{CH}_{2}\right), 104.9(\mathrm{CH}), 113.4(\mathrm{CH}), 114.5(\mathrm{CH}), 116.2(\mathrm{CH}), 120.3$ (Cq), $124.7(\mathrm{CH}), 126.3(\mathrm{Cq}), 126.4(\mathrm{Cq}), 126.5(\mathrm{Cq}), 127.2(\mathrm{CH}), 127.6(\mathrm{Cq}+2 \mathrm{CH}), 127.8(\mathrm{Cq}), 128.0$ $(\mathrm{Cq}+2 \mathrm{CH}), 128.3(\mathrm{CH}), 128.4(\mathrm{CH}), 128.5(2 \mathrm{CH}), 129.6(\mathrm{CH}), 129.7(2 \mathrm{CH}), 131.6(\mathrm{Cq}), 132.8(\mathrm{CH})$, $134.5(\mathrm{CH}), 135.7(\mathrm{Cq}), 136.4(\mathrm{Cq}), 137.8(\mathrm{Cq}), 151.9(\mathrm{CH}), 155.5(\mathrm{Cq}), 170.4(\mathrm{CO}), 173.4(\mathrm{CO}) ; \mathrm{MS}$ (IS) : $615(\mathrm{M}+1)^{+}$.

\section{3-(1-Benzenesulfonyl-6-benzyloxy-1H-indol-3-yl)-4-(3-hydroxy-naphthalen-2-yl)-1-methyl-}

pyrrole-2,5-dione (22). $\mathrm{Mp} 236^{\circ} \mathrm{C} ; \mathrm{Rf}$ (petroleum ether7/EtOAc 3) : $0.55 ; \mathrm{R}(\mathrm{KBr}) \vee 3452,3260$, 3168, 2876, 1681, 1452, 1273, $1002 ;{ }^{1} \mathrm{H}$ NMR $\left(\mathrm{CDCl}_{3}\right): 3.22(\mathrm{~s}, 3 \mathrm{H}), 5.02(\mathrm{~s}, 2 \mathrm{H}), 6.28(\mathrm{~d}, 1 \mathrm{H}, J=$ 8.7), 6.37 (dd, $1 \mathrm{H}, J=2.2, J=9.0), 7.23(\mathrm{~d}, 1 \mathrm{H}, J=7.0), 7.27-7.7 .43(\mathrm{~m}, 12 \mathrm{H}), 7.51-7.75(\mathrm{~m}, 3 \mathrm{H}), 7.75$ $(\mathrm{d}, 2 \mathrm{H}, J=8.7), 8.18\left(\mathrm{~s}, 1 \mathrm{H}\right.$, exchangeable $\left.\mathrm{D}_{2} \mathrm{O}\right) ;{ }^{13} \mathrm{C} \mathrm{NMR}\left(\mathrm{CDCl}_{3}\right): 24.5\left(\mathrm{CH}_{3}\right), 70.2\left(\mathrm{CH}_{2}\right), 98.8$ $(\mathrm{CH}), 111.7(\mathrm{Cq}), 113.4(\mathrm{CH}), 113.8(\mathrm{CH}), 119.7(\mathrm{Cq}), 120.6(\mathrm{Cq}), 122.6(\mathrm{CH}), 124.1(\mathrm{CH}), 126.2$ $(\mathrm{CH}), 126.9(2 \mathrm{CH}), 127.3(\mathrm{Cq}+2 \mathrm{CH}), 127.7(\mathrm{CH}), 128.0(\mathrm{CH}), 128.2(\mathrm{CH}), 128.6(\mathrm{CH}), 129.3(2 \mathrm{CH})$, 
$129.4(2 \mathrm{CH}), 131.4(\mathrm{Cq}), 132.3(\mathrm{CH}), 133.5(\mathrm{Cq}), 134.1(\mathrm{Cq}), 135.3(\mathrm{Cq}), 135.9(\mathrm{CH}), 136.7(\mathrm{Cq}), 137.4$ (Cq), $151.5(\mathrm{Cq}), 156.9(\mathrm{Cq}), 170.2(\mathrm{CO}), 172.9(\mathrm{CO})$; MS (IS) $615(\mathrm{M}+1)^{+}$.

\section{3-(1-Benzenesulfonyl-1H-pyrrolo[2,3-b]pyridin-3-yl)-4-(3-hydroxy-naphthalen-2-yl)-1-methyl-}

pyrrole-2,5-dione (23). Mp $230{ }^{\circ} \mathrm{C}$; Rf (petroleum ether/EtOAc 6/4) : 0.64 ; IR (KBr) v. 3458, 3230, 2934, 1707, 1449, 1175, $\left.990 ;{ }^{1} \mathrm{H} \mathrm{NMR}_{\left(\mathrm{CDCl}_{3}\right)}\right) 3.22$ (s, 3H), 6.61 (m, 2H), 7.22-7.57 (m, 10H), 8.24 (m, 3H), $7.49(\mathrm{~s}, 1 \mathrm{H}) ;{ }^{13} \mathrm{C} \mathrm{NMR}\left(\mathrm{CDCl}_{3}\right): 25.0\left(\mathrm{CH}_{3}\right), 107.2(\mathrm{Cq}), 112.3(\mathrm{CH}), 117.9(\mathrm{Cq}), 118.5(\mathrm{CH})$, $123.3(\mathrm{CH}), 125.1(\mathrm{CH}), 126.9(\mathrm{Cq}), 127.1(\mathrm{CH}), 127.3(2 \mathrm{CH}), 128.1(2 \mathrm{CH}), 129.1(\mathrm{Cq}), 129.5(\mathrm{CH}+$ Cq), $130.2(\mathrm{Cq}), 131.3(\mathrm{CH}), 131.9(\mathrm{Cq}), 133.4(\mathrm{CH}), 134.4(\mathrm{Cq}), 136.6(\mathrm{Cq}), 141.8(\mathrm{CH}), 144.3(\mathrm{CH})$, $150.3(\mathrm{CH}+\mathrm{Cq}), 168.9(\mathrm{CO}), 171.7(\mathrm{CO}) ; \mathrm{MS}(\mathrm{IS}) 510(\mathrm{M}+1)^{+}$.

\section{Trifluoromethanesulfonic acid 3-[4-(1-benzenesulfonyl-5-benzyloxy-1H-indol-3-yl)-1-methyl-} 2,5-dioxo-2,5-dihydro-1H-pyrrol-3-yl]-naphthalen-2-yl ester (24). Mp $188{ }^{\circ} \mathrm{C}$; Rf (petroleum ether 7/EtOAc 3) : 0.48 ; IR (KBr): v 3153, 3034, 1672, 1706, 1438, 1225, 1155, 990, 725 ; \%). ${ }^{1} \mathrm{H}$ NMR $\left(\mathrm{CDCl}_{3},\right)$ : $3.27(\mathrm{~s}, 3 \mathrm{H}), 5.89(\mathrm{~s}, 2 \mathrm{H}), 6.76-6.81(\mathrm{~m}, 3 \mathrm{H}), 7.22-7.27(\mathrm{~m}, 4 \mathrm{H}), 7.49-7.65(\mathrm{~m}, 6 \mathrm{H}), 7.82-$ $7.97(\mathrm{~m}, 5 \mathrm{H}), 8.23(\mathrm{~s}, 1 \mathrm{H}), 8.44(\mathrm{~s}, 1 \mathrm{H}) ;{ }^{13} \mathrm{C} \mathrm{NMR}\left(\mathrm{CDCl}_{3},\right)$ : $24.6\left(\mathrm{CH}_{3}\right), 69.1(\mathrm{Cq}), 103.4(\mathrm{CH}), 111.0$ $(\mathrm{Cq}), 114.4(\mathrm{CH}), 115.6(\mathrm{CH}), 120.1(\mathrm{Cq}), 120.8(\mathrm{CH}), 122.3(\mathrm{Cq}), 127.0(\mathrm{Cq}+3 \mathrm{CH}), 127.5(\mathrm{Cq})$, $127.7(2 \mathrm{CH}), 128.1(2 \mathrm{CH}+\mathrm{Cq}), 128.4(2 \mathrm{CH}), 128.8(\mathrm{CH}), 129.2(\mathrm{CH}), 129.4(2 \mathrm{CH}), 131.5(\mathrm{CH}), 131.8$ $(\mathrm{CH}), 132.8(\mathrm{CH}), 133.2(\mathrm{Cq}), 133.4(\mathrm{Cq}), 134.1(\mathrm{CH}), 135.8(\mathrm{Cq}), 137.5(\mathrm{Cq}), 144.9(\mathrm{Cq}), 155.3(\mathrm{Cq})$, $169.6(\mathrm{CO}), 169.9(\mathrm{CO})$; MS (IS) $747(\mathrm{M}+1)^{+}$.

\section{Trifluoromethanesulfonic acid 3-[4-(1-benzenesulfonyl-6-benzyloxy-1H-indol-3-yl)-1-methyl-} 2,5-dioxo-2,5-dihydro-1H-pyrrol-3-yl]-naphthalen-2-yl ester (25). Mp $241{ }^{\circ} \mathrm{C}$; $\mathrm{Rf}$ (petroleum ether/EtOAc 7/3) : 0.71; IR (KBr): v. 3165, 3082, 2918, 1702, 1594, 882, 818, $748 ;{ }^{1} \mathrm{H} \mathrm{NMR}\left(\mathrm{CDCl}_{3},\right)$ : $3.25(\mathrm{~s}, 3 \mathrm{H}), 5.04(\mathrm{~s}, 2 \mathrm{H}), 6.31(\mathrm{~d}, 1 \mathrm{H}, J=8.7), 6.44(\mathrm{dd}, 1 \mathrm{H},=2.2, J=9.0), 7.34-7.43(\mathrm{~m}, 6 \mathrm{H}), 5.51-$ $7.61(\mathrm{~m}, 6 \mathrm{H}), 7.79-7.92(\mathrm{~m}, 4 \mathrm{H}), 8.17(\mathrm{~s}, 1 \mathrm{H}), 8.29(\mathrm{~s}, 1 \mathrm{H}) ;{ }^{13} \mathrm{C} \mathrm{NMR}\left(\mathrm{CDCl}_{3}\right): 24.5\left(\mathrm{CH}_{3}\right), 60.3(\mathrm{Cq})$, $70.2\left(\mathrm{CH}_{2}\right), 98.7(\mathrm{CH}), 111.7(\mathrm{Cq}), 113.3(\mathrm{CH}), 115.7(\mathrm{Cq}), 120.1(\mathrm{CH}), 121.1(\mathrm{Cq}), 121.6(\mathrm{CH}), 121.7 \mathrm{~S} 7$ 
$(\mathrm{Cq}), 126.9(2 \mathrm{CH}), 127.3(2 \mathrm{CH}), 127.7(2 \mathrm{CH}), 127.9(\mathrm{CH}), 128.4(\mathrm{CH}), 128.5(2 \mathrm{CH}), 128.7(\mathrm{CH})$, $129.3(2 \mathrm{CH}), 129.6(\mathrm{CH}), 131.7(\mathrm{Cq}), 133.0(\mathrm{CH}), 133.2(\mathrm{Cq}), 134.1(\mathrm{CH}), 133.4(\mathrm{Cq}), 135.7(\mathrm{Cq})$, $136.5(\mathrm{Cq}), 137.3(\mathrm{Cq}), 144.6(\mathrm{Cq}), 156.9(\mathrm{Cq}), 169.6(\mathrm{CO}), 169.9(\mathrm{CO})$; MS (IS) $747(\mathrm{M}+1)^{+}$.

\section{Trifluoromethanesulfonic acid 3-[4-(1-benzenesulfonyl-1 $H$-pyrrolo[2,3-b]pyridin-3-yl)-1-methyl-} 2,5-dioxo-2,5-dihydro-1H-pyrrol-3-yl]-naphthalen-2-yl ester (26). Mp $128{ }^{\circ} \mathrm{C}$; $\mathrm{Rf}$ (petroleum ether/EtOAc 6/4) : $0.72 ; \mathrm{IR}(\mathrm{KBr}): v 3172,2926,1710,1519,1448,1385,1219,832,754,685 ;{ }^{1} \mathrm{H}$ NMR $\left(\mathrm{CDCl}_{3},\right)$ : $3.25(\mathrm{~s}, 3 \mathrm{H}), 6.78(\mathrm{~m}, 2 \mathrm{H}), 7.27-7.66(\mathrm{~m}, 6 \mathrm{H}), 7.84-7.90(\mathrm{~m}, 2 \mathrm{H}), 8.12(\mathrm{~s}, 1 \mathrm{H}), 8.19(\mathrm{~d}$, $2 \mathrm{H}, J=9.0), 8.28(\mathrm{dd}, 1 \mathrm{H}, J=1.5, J=4.8), 8.45(\mathrm{~s}, 1 \mathrm{H}) ;{ }^{13} \mathrm{C} \mathrm{NMR}\left(\mathrm{CDCl}_{3},\right): 24.1\left(\mathrm{CH}_{3}\right), 107.5(\mathrm{Cq})$, $114.7(\mathrm{Cq}), 118.5(\mathrm{CH}), 119.3(\mathrm{Cq}), 119.8(\mathrm{Cq}), 120.8(\mathrm{CH}), 127.3(2 \mathrm{CH}), 127.6(\mathrm{CH}), 127.7(\mathrm{CH})$, $128.0(\mathrm{CH}), 128.4(2 \mathrm{CH}), 128.5(\mathrm{CH}), 129.2(\mathrm{CH}), 129.9(\mathrm{CH}), 131.2(\mathrm{CH}), 132.5(\mathrm{Cq}), 132.7(\mathrm{Cq})$, $132.8(\mathrm{Cq}), 133.8(\mathrm{CH}+\mathrm{Cq}), 136.9(\mathrm{Cq}), 143.7(\mathrm{Cq}), 144.9(\mathrm{CH}), 146.4(\mathrm{Cq}), 168.9(\mathrm{CO}), 169.0(\mathrm{CO})$; MS (IS) : $642(\mathrm{M}+1)^{+}$.

3-(Benzyloxy)-6-methylnaphtho[2,3-a]pyrrolo[3,4-c]carbazole-5,7(6H,14H)-dione (27). Mp 232 ${ }^{\circ} \mathrm{C}$ (dec.) ; Rf (petroleum ether/EtOAc 4/6) 0.57; IR (KBr): v 2924, 1754, 1685, 1478, 1381, 1226, 891 ; ${ }^{1} \mathrm{H}$ NMR (DMSO- $\left.d_{6}, 80^{\circ} \mathrm{C}\right): 2.96(\mathrm{~s}, 3 \mathrm{H}), 5.15(\mathrm{~s}, 2 \mathrm{H}), 7.11(\mathrm{dd}, 1 \mathrm{H}, J=2.4, J=8.8), 7.37-7.58(\mathrm{~m}$, $8 \mathrm{H}), 7.97(\mathrm{~m}, 2 \mathrm{H}), 8.30(\mathrm{~s}, 1 \mathrm{H}), 8.94(\mathrm{~s}, 1 \mathrm{H}), 9.25(\mathrm{~s}, 1 \mathrm{H}), 12.79\left(\mathrm{~s}, 1 \mathrm{H}\right.$, exchangeable $\left.\mathrm{D}_{2} \mathrm{O}\right) ;{ }^{13} \mathrm{C}$ NMR (DMSO- $\left.d_{6}, 80^{\circ} \mathrm{C},\right): 23.8\left(\mathrm{CH}_{3}\right), 70.8\left(\mathrm{CH}_{2}\right), 108.0(\mathrm{CH}), 110.3(\mathrm{Cq}), 112.8(\mathrm{CH}), 116.4(\mathrm{Cq}), 119.6$ (Cq), $121.5(\mathrm{CH}), 122.9(\mathrm{Cq}), 124.0(\mathrm{Cq}), 124.5(\mathrm{CH}), 123.1(\mathrm{Cq}), 123.5(\mathrm{Cq}), 126.9(2 \mathrm{CH}), 127.0$ $(\mathrm{CH}), 127.1(2 \mathrm{CH}+\mathrm{Cq}), 127.2(\mathrm{CH}), 128.2(\mathrm{CH}), 128.9(\mathrm{Cq}), 131.9(\mathrm{CH}), 132.4(\mathrm{CH}), 134.9(\mathrm{CH})$, $138.2(\mathrm{Cq}), 140.9(\mathrm{Cq}), 154.2(\mathrm{Cq}), 169.5(\mathrm{CO}), 170.3(\mathrm{CO})$; MS (IS) : $457(\mathrm{M}+\mathrm{H})^{+}$.

2-(Benzyloxy)-6-methylnaphtho[2,3-a]pyrrolo[3,4-c]carbazole-5,7(6H,14H)-dione (28). Mp 193 ${ }^{\circ} \mathrm{C}$ (dec.) ; Rf (petroleum ether/EtOAc 7/3) 0.83; IR (KBr): $v$ 3252, 2940, 2854, 1702, 1474, 1388, $1174,804,733 ;{ }^{1} \mathrm{H}$ NMR (DMSO- $\left.d_{6}\right): 2.86(\mathrm{~s}, 3 \mathrm{H}), 5.21(\mathrm{~s}, 2 \mathrm{H}), 6.95(\mathrm{dd}, 1 \mathrm{H}, J=2.0, J=8.7), 7.04$ (s, 1H), 7.37-7.57 (m, 7H), $7.97(\mathrm{~d}, 1 \mathrm{H}, J=7.0), 8.05(\mathrm{~d}, 1 \mathrm{H}, J=8.7), 8.52(\mathrm{~d}, 1 \mathrm{H}, J=8.7), 8.80(\mathrm{~s}, \mathrm{~s} 8$ 
1H), $9.20(\mathrm{~s}, 1 \mathrm{H}), 12.70\left(\mathrm{~s}, 1 \mathrm{H}\right.$, exchangeable $\left.\mathrm{D}_{2} \mathrm{O}\right) ;{ }^{13} \mathrm{C}$ NMR (DMSO-d $): 23.7\left(\mathrm{CH}_{3}\right), 70.3\left(\mathrm{CH}_{2}\right)$, $96.5(\mathrm{CH}), 110.3(\mathrm{Cq}), 111.4(\mathrm{CH}), 116.4(\mathrm{Cq}), 118.0(\mathrm{Cq}), 121.3(\mathrm{CH}+\mathrm{Cq}), 123.6(\mathrm{Cq}), 124.4(\mathrm{CH})$, $124.9(\mathrm{CH}), 126.8(\mathrm{CH}), 127.3(\mathrm{CH}), 128.5(2 \mathrm{CH}+\mathrm{Cq}), 128.6(\mathrm{CH}), 128.7(\mathrm{CH}), 129.3(2 \mathrm{CH}+\mathrm{Cq})$, $131.9(\mathrm{Cq}), 132.1(\mathrm{Cq}), 138.1(\mathrm{Cq}), 140.2(\mathrm{Cq}), 141.1(\mathrm{CH}), 158.2(\mathrm{Cq}), 169.5(\mathrm{CO}), 170.4(\mathrm{CO})$; MS (IS) : $457(\mathrm{M}+\mathrm{H})^{+}$.

\section{3-(Hydroxy)-6-methylnaphtho[2,3-a]pyrrolo[3,4-c $]$ carbazole-5,7(6H,14H)-dione (29). Mp $214{ }^{\circ} \mathrm{C}$}

; Rf (petroleum ether/EtOAc 4/6) : 0.55 ; IR (KBr): v 3666, 3268, 2924, 1683, 1474, 1382, 1224, 784 ;

${ }^{1} \mathrm{H}$ NMR $\left(\mathrm{DMSO}-d_{6}\right): 3.13(\mathrm{~s}, 3 \mathrm{H}), 6.95(\mathrm{dd}, 1 \mathrm{H}, J=2.4, J=8.8), 7.55(\mathrm{~d}, 1 \mathrm{H}, J=9.5), 7.64-7.66(\mathrm{~m}$, 2H), $8.12(\mathrm{~d}, 1 \mathrm{H}, J=4.1), 8.21(\mathrm{~d}, 1 \mathrm{H}, J=4.1), 8.31(\mathrm{~s}, 1 \mathrm{H}), 9.20(\mathrm{~s}, 1 \mathrm{H}), 9.29(\mathrm{~s}, 1 \mathrm{H}), 9.52(\mathrm{~s}, 1 \mathrm{H}$, exchangeable $\left.\mathrm{D}_{2} \mathrm{O}\right), 12.94\left(\mathrm{~s}, 1 \mathrm{H}\right.$, exchangeable $\left.\mathrm{D}_{2} \mathrm{O}\right) ;{ }^{13} \mathrm{C}$ NMR (DMSO- $\left.d_{6}\right): 21.8\left(\mathrm{CH}_{3}\right), 106.5(\mathrm{Cq})$, $107.8(\mathrm{CH}), 110.7(\mathrm{Cq}), 114.1(\mathrm{CH}), 115.4(\mathrm{CH}), 119.2(\mathrm{Cq}), 121.1(\mathrm{CH}), 122.3(\mathrm{Cq}), 124.0(\mathrm{CH})$, $124.9(\mathrm{Cq}), 125.3(\mathrm{Cq}), 126.5(\mathrm{Cq}), 127.1(\mathrm{CH}), 127.8(\mathrm{CH}), 128.8(\mathrm{CH}), 129.7(\mathrm{Cq}), 130.2(\mathrm{CH}), 131.8$ $(\mathrm{Cq}), 138.7(\mathrm{Cq}), 150.7(\mathrm{Cq}), 167.6(\mathrm{CO}), 168.5(\mathrm{CO}) . \mathrm{MS}(\mathrm{IS}): 367(\mathrm{M}+\mathrm{H}){ }^{+} .{ }^{1} \mathrm{H}$ NMR (DMSO- $\left.d_{6}\right)$ : $3.06(\mathrm{~s}, 3 \mathrm{H}), 6.95(\mathrm{dd}, 1 \mathrm{H}, J=1.8, J=8.5), 7.08(\mathrm{~d}, 1 \mathrm{H}, J=1.8), 7.61-7.67(\mathrm{~m}, 2 \mathrm{H}), 8.03(\mathrm{~d}, 1 \mathrm{H}, J=$ 9.1), $8.05(\mathrm{dd}, 1 \mathrm{H}, J=2.5, J=9.1), 8.59(\mathrm{~d}, 1 \mathrm{H}, J=8.5), 9.01(\mathrm{~s}, 1 \mathrm{H}), 9.38(\mathrm{~s}, 1 \mathrm{H}), 9.75(\mathrm{~s}, 1 \mathrm{H}$, exchangeable $\left.\mathrm{D}_{2} \mathrm{O}\right), 12.76\left(\mathrm{~s}, 1 \mathrm{H}\right.$, exchangeable $\left.\mathrm{D}_{2} \mathrm{O}\right) ;{ }^{13} \mathrm{C}$ NMR $\left(\mathrm{DMSO}-d_{6}\right): 24.5\left(\mathrm{CH}_{3}\right), 97.9(\mathrm{CH})$, $111.1(\mathrm{CH}), 116.4(\mathrm{Cq}), 112.0(\mathrm{Cq}), 115.6(\mathrm{Cq}), 118.2(\mathrm{Cq}), 121.6(\mathrm{CH}), 121.7(\mathrm{CH}), 124.0(\mathrm{Cq}), 125.3$ $(\mathrm{CH}), 127.3(\mathrm{CH}), 127.7(\mathrm{CH}), 128.9(\mathrm{CH}), 129.2(\mathrm{Cq}), 129.5(\mathrm{CH}), 132.2(\mathrm{Cq}), 132.4(\mathrm{Cq}), 140.5(\mathrm{Cq})$, $142.0(\mathrm{Cq}), 157.7(\mathrm{Cq}), 170.1(\mathrm{CO}), 171.0(\mathrm{CO}) . \mathrm{MS}(\mathrm{IS}): 367(\mathrm{M}+\mathrm{H})^{+}$

\section{2-(Hydroxy)-6-methylnaphtho[2,3-a]pyrrolo[3,4-c $]$ carbazole-5,7(6H,14H)-dione (30). Mp $232{ }^{\circ} \mathrm{C}$}

; Rf (petroleum ether/EtOAc 1/9) : 0.58 ; IR (KBr): v 3424, 3054, 2932, 2932, 2846, 1744, 1688, 1379, 1157, 1050, $733 ;{ }^{1} \mathrm{H}$ NMR (DMSO- $\left.d_{6}\right): 3.06(\mathrm{~s}, 3 \mathrm{H}), 6.95(\mathrm{dd}, 1 \mathrm{H}, J=1.8, J=8.5), 7.08(\mathrm{~d}, 1 \mathrm{H}, J=$ 1.8), 7.61-7.67 (m, 2H), $8.03(\mathrm{~d}, 1 \mathrm{H}, J=9.1), 8.05(\mathrm{dd}, 1 \mathrm{H}, J=2.5, J=9.1), 8.59(\mathrm{~d}, 1 \mathrm{H}, J=8.5), 9.01$ $(\mathrm{s}, 1 \mathrm{H}), 9.38(\mathrm{~s}, 1 \mathrm{H}), 9.75\left(\mathrm{~s}, 1 \mathrm{H}\right.$, exchangeable $\left.\mathrm{D}_{2} \mathrm{O}\right), 12.76\left(\mathrm{~s}, 1 \mathrm{H}\right.$, exchangeable $\left.\mathrm{D}_{2} \mathrm{O}\right) ;{ }^{13} \mathrm{C}$ NMR $\left(\mathrm{DMSO}-d_{6}\right)$ : $24.5\left(\mathrm{CH}_{3}\right), 97.9(\mathrm{CH}), 111.1(\mathrm{CH}), 116.4(\mathrm{Cq}), 112.0(\mathrm{Cq}), 115.6(\mathrm{Cq}), 118.2(\mathrm{Cq}), 121.6 \mathrm{~S} 9$ 
$(\mathrm{CH}), 121.7(\mathrm{CH}), 124.0(\mathrm{Cq}), 125.3(\mathrm{CH}), 127.3(\mathrm{CH}), 127.7(\mathrm{CH}), 128.9(\mathrm{CH}), 129.2(\mathrm{Cq}), 129.5$

(CH), $132.2(\mathrm{Cq}), 132.4(\mathrm{Cq}), 140.5(\mathrm{Cq}), 142.0(\mathrm{Cq}), 157.7(\mathrm{Cq}), 170.1(\mathrm{CO}), 171.0(\mathrm{CO})$. MS (IS) : $367(\mathrm{M}+\mathrm{H})^{+}$.

\section{6-Methyl-14-[2-(4-Morpholinyl)ethyl]-naphtho[2,3-a]pyrrolo[3,4-c]carbazole-5,7(6H,14H)-dione}

(31). Mp: $242{ }^{\circ} \mathrm{C}$; Rf (EtOAc) ): 0.45 ; IR (KBr): v 2926, 2851, 1754, 1693, 379, 1114, $754 ;{ }^{1} \mathrm{H}$ NMR $\left(\mathrm{CDCl}_{3}\right): 2.53(\mathrm{~m}, 4 \mathrm{H}), 2.77(\mathrm{t}, 2 \mathrm{H}, J=7.3), 2.91(\mathrm{~s}, 3 \mathrm{H}), 3.79(\mathrm{~m}, 4 \mathrm{H}), 4.41(\mathrm{t}, 2 \mathrm{H}, J=7.3), 7.41-7.69$ $(\mathrm{m}, 5 \mathrm{H}), 7.85(\mathrm{~m}, 1 \mathrm{H}), 8.04(\mathrm{~m}, 1 \mathrm{H}), 8.64(\mathrm{~s}, 1 \mathrm{H}), 9.05(\mathrm{~d}, 1 \mathrm{H}, J=7.7), 9.49(\mathrm{~s}, 1 \mathrm{H}) ;{ }^{13} \mathrm{C} \mathrm{NMR}$ $\left(\mathrm{CDCl}_{3}\right): 23.4\left(\mathrm{CH}_{3}\right), 44.2\left(\mathrm{CH}_{2}\right), 53.9\left(2 \mathrm{CH}_{2}\right), 56.7\left(\mathrm{CH}_{2}\right), 66.9\left(2 \mathrm{CH}_{2}\right), 108.8(\mathrm{CH}), 121.1(\mathrm{CH})$, $121.6(\mathrm{CH}), 124.6(\mathrm{Cq}), 124.8(\mathrm{CH}), 125.2(\mathrm{CH}), 125.8(\mathrm{CH}), 126.6(\mathrm{CH}), 126.7(\mathrm{Cq}), 128.2(\mathrm{Cq})$, $128.3(\mathrm{Cq}), 128.5(\mathrm{Cq}), 128.6(\mathrm{CH}), 131.2(\mathrm{Cq}), 131.5(\mathrm{Cq}), 131.8(\mathrm{Cq}), 131.9(\mathrm{CH}), 132.0(\mathrm{CH}), 137.9$ (Cq), $140.3(\mathrm{Cq}), 168.8(\mathrm{CO}), 169.8(\mathrm{CO})$; MS (IS) : $464(\mathrm{M}+\mathrm{H})^{+}$.

\section{6-Methyl-14-[2-dimethylamino)-ethyl]-naphtho[2,3-a]pyrrolo[3,4-c]carbazole-5,7(6H,14H)-}

dione (32). $\mathrm{Mp}: 222{ }^{\circ} \mathrm{C} ; \mathrm{Rf}(\mathrm{EtOAc} / \mathrm{MeOH} 9 / 1): 0.55 ; \mathrm{IR}(\mathrm{KBr}): v 2918,1696,1378,1104,757 ;{ }^{1} \mathrm{H}$ $\operatorname{NMR}\left(\mathrm{CDCl}_{3}\right): 2.40(\mathrm{~s}, 6 \mathrm{H}), 2.51(\mathrm{t}, 2 \mathrm{H}, J=8.0), 2.72(\mathrm{~s}, 3 \mathrm{H}), 4.21(\mathrm{t}, 2 \mathrm{H}, J=8.0), 7.27(\mathrm{~d}, 1 \mathrm{H}, J=$ 7.3), $7.36(\mathrm{t}, 1 \mathrm{H}, J=7.0), 7.54(\mathrm{~m}, 3 \mathrm{H}), 7.77(\mathrm{~m}, 1 \mathrm{H}), 7,93(\mathrm{~m}, 1 \mathrm{H}), 8.43(\mathrm{~s}, 1 \mathrm{H}), 8.94(\mathrm{~d}, 1 \mathrm{H}, J=7.8)$, $9.29(\mathrm{~s}, 1 \mathrm{H}) ;{ }^{13} \mathrm{C} \mathrm{NMR}\left(\mathrm{CDCl}_{3}\right): 30.7\left(\mathrm{CH}_{2}\right), 37.0\left(2 \mathrm{CH}_{3}\right), 53.4\left(\mathrm{CH}_{2}\right), 116.4(\mathrm{CH}), 118.8(\mathrm{Cq}), 126.4$ $(\mathrm{CH}), 128.5(\mathrm{CH}), 128.6(\mathrm{Cq}), 128.7(\mathrm{CH}), 129.2(\mathrm{Cq}), 132.0(\mathrm{CH}), 132.4(\mathrm{CH}), 132.7(\mathrm{CH}), 133.1$ $(\mathrm{CH}), 133.5(\mathrm{Cq}), 134.1(\mathrm{Cq}), 134.2(\mathrm{Cq}), 135.4(\mathrm{CH}), 136.0(\mathrm{CH}), 136.1(\mathrm{Cq}), 138.8(\mathrm{Cq}), 139.0(\mathrm{Cq})$, $145.4(\mathrm{Cq}), 147.8(\mathrm{Cq}), 176.3(\mathrm{CO}), 177.2(\mathrm{CO})$; MS (IS) $422(\mathrm{M}+1)^{+}$.

6-Methyl-14-allyl-naphtho[2,3-a]pyrrolo[3,4-c $]$ carbazole-5,7(6H,14H)-dione (33). Mp : $228{ }^{\circ} \mathrm{C}$; Rf (petroleum ether/EtOAc 7/3) : 0.83 ; IR (KBr): v 3046, 2932, 1702, 1396, 1261, $726 ;{ }^{1} \mathrm{H}$ NMR $\left(\mathrm{CDCl}_{3}\right): 3.10(\mathrm{~s}, 3 \mathrm{H}), 5.09(\mathrm{~d}, 1 \mathrm{H}, J=17.2), 5.32(\mathrm{~m}, 3 \mathrm{H}), 6.20-6.31(\mathrm{~m}, 1 \mathrm{H}), 7.29(\mathrm{~d}, 1 \mathrm{H}, J=7.7)$, 7.41-7.58 (m, 4H), $7.85(\mathrm{~m}, 1 \mathrm{H}), 8.02(\mathrm{~m}, 1 \mathrm{H}), 8.86(\mathrm{~s}, 1 \mathrm{H}), 9.18(\mathrm{~d}, 1 \mathrm{H}, J=7.8), 9.72(\mathrm{~s}, 1 \mathrm{H}) ;{ }^{13} \mathrm{C}$ $\operatorname{NMR}\left(\mathrm{CDCl}_{3}\right): 30.9\left(\mathrm{CH}_{3}\right), 56.3\left(\mathrm{CH}_{2}\right), 116.8(\mathrm{CH}), 119.2(\mathrm{Cq}), 125.4\left(\mathrm{CH}_{2}\right), 126.9(2 \mathrm{CH}), 128.9(\mathrm{Cq})$, 
$129.0(\mathrm{Cq}), 129.4(\mathrm{Cq}), 129.6(\mathrm{Cq}), 132.3(\mathrm{CH}), 132.8(\mathrm{CH}), 133.7(\mathrm{CH}), 133.8(\mathrm{Cq}), 134.2(2 \mathrm{CH})$, $135.9(\mathrm{CH}), 136.2(\mathrm{CH}), 136.3(\mathrm{Cq}), 139.1(\mathrm{CH}), 139.7(\mathrm{Cq}), 146.5(\mathrm{Cq}), 148.5(\mathrm{Cq}), 176.8(\mathrm{CO}), 177.8$ (CO) ; MS (IS): $391(\mathrm{M}+1)^{+}$.

\section{6-Methyl-14-(2,3-dihydroxypropyl)-naphtho[2,3-a]pyrrolo[3,4-c]carbazole-5,7(6H,14H)-dione}

(34). Mp : $168{ }^{\circ} \mathrm{C} ; \operatorname{Rf}$ (petroleum ether/EtOAc 1/9) : $0.45 ; \mathrm{IR}(\mathrm{KBr}): v 3418,2925,2854,1753,1697$, 1381, 1025, $750 ;{ }^{1} \mathrm{H}$ NMR (DMSO- $\left.d_{6}\right): 2.82(\mathrm{~s}, 3 \mathrm{H}), 3.64(\mathrm{~m}, 2 \mathrm{H}), 4.20\left(\mathrm{~m}, 1 \mathrm{H}\right.$, exchangeable $\left.\mathrm{D}_{2} \mathrm{O}\right)$, $4.71\left(\mathrm{~m}, 1 \mathrm{H}\right.$, exchangeable $\left.\mathrm{D}_{2} \mathrm{O}\right), 5.11(\mathrm{~d}, 1 \mathrm{H}, J=15.0), 5.20(\mathrm{~d}, 1 \mathrm{H}, J=15.0), 5.38(\mathrm{~m}, 1 \mathrm{H}), 7.36(\mathrm{t}$, $1 \mathrm{H}, J=7.5), 7.54(\mathrm{t}, 1 \mathrm{H}, J=7.5), 7.61(\mathrm{~m}, 2 \mathrm{H}), 7.78(\mathrm{~d}, 1 \mathrm{H}, J=7.5) 8.11(\mathrm{~m}, 2 \mathrm{H}), 8.97(\mathrm{~d}, 1 \mathrm{H}, J=7.8)$, $9.51(\mathrm{~m}, 2 \mathrm{H}) ;{ }^{13} \mathrm{C}$ NMR (DMSO-d $): 24.2\left(\mathrm{CH}_{3}\right), 50.3\left(\mathrm{CH}_{2}\right), 64.8\left(\mathrm{CH}_{2}\right), 71.8(\mathrm{CH}), 111.7(\mathrm{Cq}), 112.4$ $(\mathrm{CH}), 119.2(\mathrm{Cq}), 121.4(\mathrm{Cq}), 122.0(\mathrm{CH}), 122.1(\mathrm{Cq}), 124.0(\mathrm{CH}), 124.2(\mathrm{CH}), 124.7(\mathrm{CH}), 125.3(\mathrm{Cq})$, $126.4(\mathrm{CH}), 127.4(\mathrm{CH}), 127.9(\mathrm{CH}), 128.8(\mathrm{CH}), 129.1(\mathrm{Cq}), 129.7(\mathrm{CH}), 131.7(\mathrm{Cq}), 132.13(\mathrm{Cq})$, $139.3(\mathrm{Cq}), 142.4(\mathrm{CO}), 169.7(\mathrm{CO}), 170.6(\mathrm{CO}) . \mathrm{MS}(\mathrm{IS}): 425(\mathrm{M}+1)^{+}$.

5H-Furo[3,4-c]naphtho[2,3-a]pyrrolo[3,4-c]carbazole-5,7(14H)-dione (35). Mp > $250{ }^{\circ} \mathrm{C} ; \mathrm{Rf}$ (petroleum/EtOAc 4/6) : 0.32 ; IR (KBr): v 3324, 3054, 1820, 1728, 1343, 1258, 1164, 1111, 881, 730 ; ${ }^{1} \mathrm{H}$ NMR (DMSO- $\left.d_{6}, 80^{\circ} \mathrm{C}\right): 7.40(\mathrm{t}, 1 \mathrm{H}, J=7.5), 7.52(\mathrm{~d}, 1 \mathrm{H}, J=7.5), 7.75(\mathrm{~m}, 3 \mathrm{H}), 8.14(\mathrm{~m}, 1 \mathrm{H})$, $8.22(\mathrm{~m}, 1 \mathrm{H}), 8.66(\mathrm{~m}, 1 \mathrm{H}), 9.30(\mathrm{~s}, 1 \mathrm{H}), 9.35(\mathrm{~s}, 1 \mathrm{H}), 13.20\left(\mathrm{~s}, 1 \mathrm{H}\right.$, exchangeable $\left.\mathrm{D}_{2} \mathrm{O}\right) ;{ }^{13} \mathrm{C}$ Dept NMR (DMSO- $\left.d_{6}, 80^{\circ} \mathrm{C}\right)$ : $113.0(\mathrm{CH}), 122.3(\mathrm{CH}), 122.8(\mathrm{CH}), 123.5(\mathrm{CH}), 125.4(\mathrm{CH}), 127.0(\mathrm{CH})$, $128.0(\mathrm{CH}), 128.4(\mathrm{CH}), 128.9(\mathrm{CH}), 129.5(\mathrm{CH})$; MS (IS) : $338(\mathrm{M}+1)^{+}$.

\section{6-[2-(Dimethylamino)ethyl]-naphtho[2,3-a]pyrrolo[3,4-c]carbazole-5,7-(6H,14H)-dione $\quad(36)$.}

$\mathrm{Mp}: 250{ }^{\circ} \mathrm{C}$ dec ; Rf (acetone) : 0.45 ; IR (KBr): $\vee 3485,3158,3060,2940,2790,1751,1694,1389$, 1004, $734 ;{ }^{1} \mathrm{H}$ NMR (DMSO- $\left.d_{6}\right): 2.48(\mathrm{~s}, 6 \mathrm{H}), 2.53(\mathrm{t}, 2 \mathrm{H}, J=6.5), 3.73(\mathrm{t}, 2 \mathrm{H}, J=6.5), 7.34(\mathrm{t}, 1 \mathrm{H}, J$ $=7.3), 7.52(\mathrm{t}, 1 \mathrm{H}, J=8.0), 7.71(\mathrm{~m}, 3 \mathrm{H}), 8.05(\mathrm{~m}, 1 \mathrm{H}), 8.14(\mathrm{~m}, 1 \mathrm{H}), 8.78(\mathrm{~d}, 1 \mathrm{H}, J=7.8), 9.14(\mathrm{~s}$,

1H), $9.42(\mathrm{~s}, 1 \mathrm{H}), 13.09\left(\mathrm{~s}, 1 \mathrm{H}\right.$, exchangeable $\left.\mathrm{D}_{2} \mathrm{O}\right) ;{ }^{13} \mathrm{C}$ NMR (DMSO- $\left.d_{6}\right): 25.96\left(\mathrm{CH}_{2}\right), 46.0\left(2 \mathrm{CH}_{3}\right)$, $57.7\left(\mathrm{CH}_{2}\right), 110.3(\mathrm{Cq}), 112.7(\mathrm{CH}), 118.5(\mathrm{Cq}), 121.7(\mathrm{CH}), 121.9(\mathrm{CH}), 122.3(\mathrm{Cq}), 122.4(\mathrm{Cq}), 124.3$ 
$(\mathrm{Cq}+\mathrm{CH}), 124.9(\mathrm{CH}), 126.6(\mathrm{CH}), 127.6(\mathrm{CH}), 127.8(\mathrm{CH}), 128.9(\mathrm{CH}), 129.5(\mathrm{CH}), 129.7(\mathrm{Cq})$, $132.2(\mathrm{Cq}), 132.7(\mathrm{Cq}), 140.8(\mathrm{Cq}), 140.9(\mathrm{Cq}), 169.7(\mathrm{CO}), 170.7(\mathrm{CO})$; MS (IS) : $408(\mathrm{M}+1)^{+}$.

\section{3-(Hydroxy)-6-[2-(dimethylamino)ethyl]naphtho[2,3-a]pyrrolo[3,4-c]carbazole-5,7(6H,14H)-}

dione (37). $\mathrm{Mp}>250$; $\mathrm{Rf}$ (petroleum ether/EtOAc 1/9): 0.10 ; IR (KBr): v 3464, 3923, 1696, 1676, $1385 ; \%) .{ }^{1} \mathrm{H}$ NMR (DMSO- $\left.d_{6}\right): 2.51(\mathrm{~s}, 6 \mathrm{H}), 2.60(\mathrm{t}, 2 \mathrm{H}, J=5.9), 3.76(\mathrm{t}, 2 \mathrm{H}, J=5.9), 7.01(\mathrm{dd}, 1 \mathrm{H}$, $J=2.2, J=8.5), 7.55(\mathrm{~d}, 1 \mathrm{H}, J=8.5), 7.65(\mathrm{~m}, 2 \mathrm{H}), 8.10(\mathrm{~d}, 1 \mathrm{H}, J=9.0), 8.2(\mathrm{~d}, 1 \mathrm{H}, J=9.0), 8.32(\mathrm{~d}$, $1 \mathrm{H}, J=2.2), 9.19(\mathrm{~s}, 1 \mathrm{H}), 9.29(\mathrm{~s}, 1 \mathrm{H}), 9.51\left(\mathrm{~s}, 1 \mathrm{H}\right.$, exchangeable $\left.\mathrm{D}_{2} \mathrm{O}\right), 12.72(\mathrm{~s}, 1 \mathrm{H}$, exchangeable $\left.\mathrm{D}_{2} \mathrm{O}\right) ;{ }^{13} \mathrm{C}$ NMR $\left(\mathrm{DMSO}-d_{6}\right)$ : $35.6\left(\mathrm{CH}_{2}\right), 45.70\left(2 \mathrm{CH}_{3}\right), 57.5\left(\mathrm{CH}_{2}\right), 97.5(\mathrm{CH}), 110.7(\mathrm{Cq}), 111.6$ $(\mathrm{CH}), 115.1(\mathrm{Cq}), 117.4(\mathrm{Cq}), 121.3(\mathrm{Cq}+\mathrm{CH}), 123.5(\mathrm{Cq}), 124.4(\mathrm{CH}), 125.0(\mathrm{CH}), 126.8(\mathrm{Cq}), 127.2$ $(\mathrm{CH}), 128.5(\mathrm{CH}), 128.8(\mathrm{CH}), 129.1(\mathrm{CH}), 131.8(\mathrm{Cq}), 132.0(\mathrm{Cq}), 140.1(\mathrm{Cq}), 141.6(\mathrm{Cq}), 157.3(\mathrm{Cq})$, $169.5(\mathrm{CO}), 170.3(\mathrm{CO}) . \mathrm{MS}(\mathrm{IS}): 424(\mathrm{M}+1)^{+}$.

\section{2-(Hydroxy)-6-[2-(dimethylamino)ethyl]naphtho[2,3-a]pyrrolo[3,4-c]carbazole-5,7(6H,14H)-}

dione (38). $\mathrm{Mp}: 252{ }^{\circ} \mathrm{C}$; Rf (acetone) : 0.38 ; IR (KBr): v 3438, 3282, 2960, 2832, 2790, 1758, 1708, 1388, 1005, 826, $733 ;{ }^{1} \mathrm{H}$ NMR (DMSO-d $): 2.17(\mathrm{~s}, 6 \mathrm{H}), 2.48(\mathrm{~m}, 2 \mathrm{H}), 3.57(\mathrm{~m}, 2 \mathrm{H}), 6.81(\mathrm{~d}, 1 \mathrm{H}, J=$ 8.0), $7.00(\mathrm{~s}, 1 \mathrm{H}), 7.53(\mathrm{~m}, 2 \mathrm{H}), 7.93(\mathrm{~d}, 1 \mathrm{H}, J=8.0), 8.03(\mathrm{~d}, 1 \mathrm{H}, J=8.0), 8.50(\mathrm{~d}, 1 \mathrm{H}, J=8.0), 8.88$ $(\mathrm{s}, 1 \mathrm{H}), 9.25(\mathrm{~s}, 1 \mathrm{H}), 9.69\left(\mathrm{~s}, 1 \mathrm{H}\right.$, exchangeable $\left.\mathrm{D}_{2} \mathrm{O}\right), 12.64\left(\mathrm{~s}, 1 \mathrm{H}\right.$, exchangeable $\left.\mathrm{D}_{2} \mathrm{O}\right) ;{ }^{13} \mathrm{C}$ NMR $\left(\mathrm{DMSO}-d_{6}\right)$ : $35.9\left(\mathrm{CH}_{2}\right), 46.0\left(2 \mathrm{CH}_{3}\right), 57.7\left(\mathrm{CH}_{2}\right), 97.5(\mathrm{CH}), 110.7(\mathrm{Cq}), 111.6(\mathrm{CH}), 115.1(\mathrm{Cq})$, $117.4(\mathrm{Cq}), 121.3(\mathrm{Cq}+\mathrm{CH}), 123.5(\mathrm{Cq}), 124.4(\mathrm{CH}), 125.0(\mathrm{CH}), 126.8(\mathrm{Cq}), 127.2(\mathrm{CH}), 128.5(\mathrm{CH})$, $128.8(\mathrm{CH}), 129.1(\mathrm{CH}), 131.8(\mathrm{Cq}), 132.0(\mathrm{Cq}), 140.1(\mathrm{Cq}), 141.6(\mathrm{Cq}), 157.3(\mathrm{Cq}), 169.5(\mathrm{CO}), 170.3$ (CO) ; MS (IS) : $424(\mathrm{M}+1)^{+}$.

\section{3-(Hydroxy)-6-[3-(dimethylamino)propyl]naphtho[2,3-a]pyrrolo[3,4-c]carbazole-5,7(6H,14H)-}

dione (39). $\mathrm{Mp}:>250{ }^{\circ} \mathrm{C}$; $\mathrm{Rf}$ (acetone) 0.11 ; IR (KBr): $y$ 3422, 3218, 1923, 1749, 1696, 1472, 1390 , 1219, $776 ;{ }^{1} \mathrm{H}$ NMR (DMSO- $\left.d_{6}\right): 1.91(\mathrm{~m}, 2 \mathrm{H}), 2.27(\mathrm{~s}, 6 \mathrm{H}), 2.38(\mathrm{~m}, 2 \mathrm{H}), 3.76(\mathrm{~m}, 2 \mathrm{H}), 6.95(\mathrm{dd}, 1 \mathrm{H}$, $J=1.8, J=8.5), 7.08(\mathrm{~d}, 1 \mathrm{H}, J=1.8), 7.67(\mathrm{~m}, 2 \mathrm{H}), 8.03(\mathrm{~d}, 1 \mathrm{H}, J=9.1), 8.05(\mathrm{dd}, 1 \mathrm{H}, J=2.5, J=$ 
9.1), $8.59(\mathrm{~d}, 1 \mathrm{H}, J=8.5), 9.01(\mathrm{~s}, 1 \mathrm{H}), 9.38(\mathrm{~s}, 1 \mathrm{H}), 9.75\left(\mathrm{~s}, 1 \mathrm{H}\right.$, exchangeable $\left.\mathrm{D}_{2} \mathrm{O}\right), 12.76(\mathrm{~s}, 1 \mathrm{H}$, exchangeable $\left.\mathrm{D}_{2} \mathrm{O}\right) ;{ }^{13} \mathrm{C}$ NMR (DMSO- $\left.d_{6}\right): 26.0\left(\mathrm{CH}_{2}\right), 35.5\left(\mathrm{CH}_{2}\right), 44.7\left(2 \mathrm{CH}_{3}\right), 56.4\left(\mathrm{CH}_{2}\right), 107.3$ $(\mathrm{Cq}), 108.7(\mathrm{CH}), 111.7(\mathrm{CH}), 115.0(\mathrm{CH}), 116.2(\mathrm{Cq}), 120.6(\mathrm{Cq}+\mathrm{CH}), 121.9(\mathrm{Cq}), 123.0(\mathrm{Cq}), 123.4$ $(\mathrm{CH}), 126.0(\mathrm{CH}), 126.3(\mathrm{Cq}), 127.4(\mathrm{CH}), 128.0(\mathrm{CH}), 128.9(\mathrm{CH}), 130.7(\mathrm{Cq}), 131.2(\mathrm{Cq}), 132.7(\mathrm{Cq})$, $139.8(\mathrm{Cq}), 151.6(\mathrm{Cq}), 168.6(\mathrm{CO}), 169.5$ (CO) ; MS (IS) : $438(\mathrm{M}+1)^{+} .2$ 2-(Hydroxy)-6-[3(dimethylamino)propyl]naphtho[2,3-a]pyrrolo[3,4-c]carbazole-5,7(6H,14H)-dione (40). Mp : > 250 ${ }^{\circ} \mathrm{C}$; Rf (acetone) : 0.21 ; IR (KBr): y 3431, 3184, 2923, 2854, 1749, 1695, 1641, 1591, 1385, 1158, 735 ; ${ }^{1} \mathrm{H}$ NMR (DMSO- $\left.d_{6}\right) 1.82(\mathrm{~m}, 2 \mathrm{H}), 2.59(\mathrm{~m}, 6 \mathrm{H}), 3.30(\mathrm{~m}, 2 \mathrm{H}), 3.72(\mathrm{~m}, 2 \mathrm{H}), 6.86(\mathrm{~d}, 1 \mathrm{H}, J=8.8)$, $7.11(\mathrm{~s}, 1 \mathrm{H}), 7.64(\mathrm{~m}, 2 \mathrm{H}), 8.10(\mathrm{~m}, 2 \mathrm{H}), 8.66(\mathrm{~d}, 1 \mathrm{H}, J=8.5), 9.17(\mathrm{~s}, 1 \mathrm{H}), 9.53(\mathrm{~s}, 1 \mathrm{H}), 9.76(\mathrm{~s}, 1 \mathrm{H}$, exchangeable $\left.\mathrm{D}_{2} \mathrm{O}\right), 12.94\left(\mathrm{~s}, 1 \mathrm{H}\right.$, exchangeable $\left.\mathrm{D}_{2} \mathrm{O}\right) ;{ }^{13} \mathrm{C}$ NMR $\left(\right.$ DMSO- $\left.d_{6}\right): 28.4\left(\mathrm{CH}_{2}\right), 38.1\left(\mathrm{CH}_{2}\right)$, $47.0\left(2 \mathrm{CH}_{3}\right), 58.8\left(\mathrm{CH}_{2}\right), 97.9(\mathrm{Cq}), 111.2(\mathrm{Cq}), 112.1(\mathrm{CH}), 115.5(\mathrm{Cq}), 118.1(\mathrm{Cq}), 121.8(\mathrm{CH}), 121.9$ $(\mathrm{Cq}), 124.1(\mathrm{Cq}), 124.9(\mathrm{CH}), 125.3(\mathrm{CH}), 127.4(\mathrm{CH}), 127.8(\mathrm{CH}), 129.3(\mathrm{Cq}), 128.9(\mathrm{CH}), 129.6$ (CH), $132.2(\mathrm{Cq}), 132.5(\mathrm{Cq}), 140.6(\mathrm{Cq}), 142.0(\mathrm{Cq}), 157.7(\mathrm{Cq}), 170.2(\mathrm{CO}), 171.0(\mathrm{CO})$; MS (IS) : $438(\mathrm{M}+1)^{+}$.

\section{3-(Hydroxy)-6-\{2-[(2-hydroxyethyl)amino]ethyl\}naphtho[2,3-a]pyrrolo[3,4-c]carbazole-}

5,7(6H,14H)-dione (41). Mp : $183^{\circ} \mathrm{C}$; Rf (acetone) : 0.12 ; IR (KBr): y 3410, 3288, 3946, 2862, 1758, 1702, 1466, 1402, 1274, $1232 ;{ }^{1} \mathrm{H}$ NMR (DMSO- $\left.d_{6}\right) 2.62(\mathrm{t}, 2 \mathrm{H}, J=5.7), 2.85(\mathrm{t}, 2 \mathrm{H}, J=5.7), 3.34(\mathrm{~s}$, $1 \mathrm{H}$, exchangeable $\left.\mathrm{D}_{2} \mathrm{O}\right), 3.40(\mathrm{t}, 2 \mathrm{H}, J=5.7), 3.73(\mathrm{t}, 2 \mathrm{H}, J=5.8), 4.47\left(\mathrm{~s}, 1 \mathrm{H}\right.$, exchangeable $\left.\mathrm{D}_{2} \mathrm{O}\right), 7.03$ $(\mathrm{dd}, 1 \mathrm{H}, J=2.4, J=8.8), 7.56(\mathrm{~d}, 1 \mathrm{H}, J=8.8), 7.63(\mathrm{~m}, 2 \mathrm{H}), 8.09-8.12(\mathrm{~m}, 2 \mathrm{H}), 8.31(\mathrm{~d}, 1 \mathrm{H}, J=2.5)$, $9.31(\mathrm{~s}, 2 \mathrm{H}), 9.52\left(\mathrm{~s}, 1 \mathrm{H}\right.$, exchangeable $\left.\mathrm{D}_{2} \mathrm{O}\right), 12.81\left(\mathrm{~s}, 1 \mathrm{H}\right.$, exchangeable $\left.\mathrm{D}_{2} \mathrm{O}\right) ;{ }^{13} \mathrm{C}$ NMR (DMSO- $d_{6}$, Dept135) : $38.2\left(\mathrm{CH}_{2}\right), 48.2\left(\mathrm{CH}_{2}\right), 57.9\left(\mathrm{CH}_{2}\right), 61.1\left(\mathrm{CH}_{2}\right), 108.8(\mathrm{CH}), 113.2(\mathrm{CH}), 116.5(\mathrm{CH}), 122.5$ (CH), $124.8(\mathrm{CH}), 127.7(\mathrm{CH}), 128.9(\mathrm{CH}), 129.5(\mathrm{CH}) ; \mathrm{MS}(\mathrm{IS}): 440(\mathrm{M}+1)^{+}$.

\section{2-(Hydroxy)-6-\{2-[(2-hydroxyethyl)amino]ethyl\}naphtho[2,3-a]pyrrolo[3,4-c]carbazole-}

5,7(6H,14H)-dione (42). $\mathrm{Mp}: 196{ }^{\circ} \mathrm{C}$; Rf (acetone) : 0.56 ; IR (KBr): y 3423, 2930, 2846, 1749, 1689, 1629, 1384 1159, 1065, $874 ;{ }^{1} \mathrm{H}$ NMR (DMSO- $\left.d_{6}\right) 2.72$ (m, 2H), 2.89 (m, 2H), 3.42 (m, 2H), 3.63 (s, S13 
$1 \mathrm{H}$, exchangeable $\left.\mathrm{D}_{2} \mathrm{O}\right), 3.77(\mathrm{~m}, 2 \mathrm{H}), 4.46\left(\mathrm{~s}, 1 \mathrm{H}\right.$, exchangeable $\left.\mathrm{D}_{2} \mathrm{O}\right), 6.87(\mathrm{~d}, 1 \mathrm{H}, J=8.8), 7.12(\mathrm{~s}$, 1H), $7.65(\mathrm{~m}, 2 \mathrm{H}), 8.12(\mathrm{~d}, 1 \mathrm{H}, J=7.8), 7.96(\mathrm{~d}, 1 \mathrm{H}, J=7.8), 8.65(\mathrm{~d}, 1 \mathrm{H}, J=8.8), 9.21(\mathrm{~s}, 1 \mathrm{H}), 9.57$ $(\mathrm{s}, 1 \mathrm{H}), 9.77\left(\mathrm{~s}, 1 \mathrm{H}\right.$, exchangeable $\left.\mathrm{D}_{2} \mathrm{O}\right), 12.81\left(\mathrm{~s}, 1 \mathrm{H}\right.$, exchangeable $\left.\mathrm{D}_{2} \mathrm{O}\right) ;{ }^{13} \mathrm{C}$ NMR $\left(\mathrm{DMSO}-d_{6}\right)$ : $37.7\left(\mathrm{CH}_{2}\right), 47.9\left(\mathrm{CH}_{2}\right), 51.5\left(\mathrm{CH}_{2}\right), 60.7\left(\mathrm{CH}_{2}\right), 97.0(\mathrm{CH}), 110.2(\mathrm{Cq}), 111.1(\mathrm{CH}), 114.5(\mathrm{Cq}), 117.1$ $(\mathrm{Cq}), 120.9(\mathrm{Cq}+\mathrm{CH}), 123.0(\mathrm{Cq}), 123.9(\mathrm{CH}), 124.3(\mathrm{CH}), 126.4(\mathrm{Cq}), 126.8(\mathrm{CH}), 128.0(\mathrm{CH}), 128.4$ $(\mathrm{CH}), 128.6(\mathrm{CH}+\mathrm{Cq}), 131.5(\mathrm{Cq}), 139.6(\mathrm{Cq}), 141.1(\mathrm{Cq}), 156.8(\mathrm{Cq}), 169.3(\mathrm{CO}), 170.1(\mathrm{CO})$; MS (IS) : $440(\mathrm{M}+1)^{+}$.

\section{3-(Hydroxy)-6-[2-hydroxy-1-(hydroxymethyl)ethyl]naphtho[2,3-a]pyrrolo[3,4-c]carbazole-}

5,7(6H,14H)-dione (43). Mp : $196{ }^{\circ} \mathrm{C}$; Rf (EtOAc) : 0.36 ; IR (KBr): y 3404, 1920, 1832, 1741, 1686, 1473, 1374, 1175, 873, $802 ;{ }^{1} \mathrm{H}$ NMR (DMSO-d $): 3.79(\mathrm{~m}, 2 \mathrm{H}), 3.94(\mathrm{~m}, 2 \mathrm{H}), 4.39(\mathrm{~m}, 1 \mathrm{H}), 4.96(\mathrm{~m}$, 2H, exchangeable $\left.\mathrm{D}_{2} \mathrm{O}\right), 7.02(\mathrm{~d}, 1 \mathrm{H}, J=8.8), 7.61(\mathrm{~m}, 3 \mathrm{H}), 8.17(\mathrm{~m}, 2 \mathrm{H}), 8.36(\mathrm{~s}, 1 \mathrm{H}), 9.29(\mathrm{~s}, 2 \mathrm{H})$, $9.60(\mathrm{~s}, 1 \mathrm{H}), 12.99\left(\mathrm{~s}, 1 \mathrm{H}\right.$, exchangeable $\left.\mathrm{D}_{2} \mathrm{O}\right) . ;{ }^{13} \mathrm{C}$ NMR (DMSO- $\left.d_{6}\right): 55.4(\mathrm{CH}), 57.96\left(2 \mathrm{CH}_{2}\right), 107.3$ $(\mathrm{CH}), 108.7$ 8(Cq), $111.7(\mathrm{CH}), 115.0(\mathrm{CH}), 116.3(\mathrm{Cq}), 120.6(\mathrm{CH}), 120.7(\mathrm{Cq}), 121.9(\mathrm{Cq}), 123.1$ $(\mathrm{CH}), 126.4(\mathrm{Cq}), 127.4(\mathrm{CH}), 127.9(\mathrm{CH}), 128.0(\mathrm{Cq}), 128.1(\mathrm{CH}), 129.0(\mathrm{CH}), 130.6(\mathrm{Cq}), 131.2(\mathrm{Cq})$, $132.7(\mathrm{Cq}), 139.7(\mathrm{Cq}), 151.6(\mathrm{Cq}), 169.5(\mathrm{CO}), 170.0(\mathrm{CO})$.; $\mathrm{MS}(\mathrm{IS})$ : $427(\mathrm{M}+1)^{+}$.

\section{2-(Hydroxy)-6-[2-hydroxy-1-(hydroxymethyl)ethyl]naphtho[2,3-a]pyrrolo[3,4-c]carbazole-}

5,7(6H,14H)-dione (44). $\mathrm{Mp}: 210^{\circ} \mathrm{C} \mathrm{dec} \mathrm{;} \mathrm{Rf} \mathrm{(petroleum} \mathrm{ether/EtOAc)} \mathrm{:} 0.35$; IR (KBr): $y$ 3452, 2928, 2848, 1762, 1685, 1375, 1158, $1022 ;{ }^{1} \mathrm{H}$ NMR (DMSO-d $) 3.78$ (m, 2H), $3.92(\mathrm{~m}, 2 \mathrm{H}), 4.36$ (m, 1H), $4.93\left(\mathrm{~m}, 2 \mathrm{H}\right.$, exchangeable $\left.\mathrm{D}_{2} \mathrm{O}\right), 6.92(\mathrm{~d}, 1 \mathrm{H}, J=8.8), 7.11(\mathrm{~s}, 1 \mathrm{H}), 7.64(\mathrm{~m}, 2 \mathrm{H}), 8.15(\mathrm{~m}, 1 \mathrm{H})$, $8.23(\mathrm{~m}, 1 \mathrm{H}), 8.69(\mathrm{~m}, 1 \mathrm{H}), 9.21(\mathrm{~s}, 1 \mathrm{H}), 9.59(\mathrm{~s}, 1 \mathrm{H}), 9.76\left(\mathrm{~s}, 1 \mathrm{H}\right.$, exchangeable $\left.\mathrm{D}_{2} \mathrm{O}\right), 13.68(\mathrm{~s}, 1 \mathrm{H}$, exchangeable $\left.\mathrm{D}_{2} \mathrm{O}\right) ;{ }^{13} \mathrm{C}$ NMR (DMSO- $\left.d_{6}\right)$ : $57.0(\mathrm{CH}), 59.5\left(2 \mathrm{CH}_{2}\right), 97.3(\mathrm{CH}), 110.4(\mathrm{Cq}), 111.4$ $(\mathrm{CH}), 114.8(\mathrm{Cq}), 117.5(\mathrm{Cq}), 121.1(\mathrm{CH}), 121.3(\mathrm{Cq}), 123.6(\mathrm{Cq}), 124.3(\mathrm{CH}), 124.7(\mathrm{CH}), 126.8(\mathrm{CH})$, $127.2(\mathrm{CH}), 128.3(\mathrm{CH}), 128.9(\mathrm{Cq}), 129.0(\mathrm{CH}), 131.6(\mathrm{Cq}), 131.9(\mathrm{Cq}), 139.9(\mathrm{Cq}), 141.4(\mathrm{Cq}), 157.1$ (Cq), 170.0 (CO), $171.0(\mathrm{CO})$; $\mathrm{MS}(\mathrm{IS}): 427(\mathrm{M}+1)^{+}$. 


\section{2-(Benzyloxy)-6-methyl-14-[2-(dimethylamino)ethyl]naphtho[2,3-a]pyrrolo[3,4-c]carbazole-}

5,7(6H,14H)-dione (45). Mp : $235^{\circ} \mathrm{C}$; Rf (EtOAc) : 0.55 ; IR (KBr): v 2918, 1715, 1696, 1381, 1273, 1104, $759 ;{ }^{1} \mathrm{H}$ NMR $\left(\mathrm{CDCl}_{3}\right): 2.42(\mathrm{~s}, 6 \mathrm{H}), 2.72(\mathrm{t}, 2 \mathrm{H}, J=7.5), 2.86(\mathrm{~s}, 3 \mathrm{H}), 4.36(\mathrm{t}, 2 \mathrm{H}, J=7.5), 5.20$ $(\mathrm{s}, 2 \mathrm{H}), 6.82(\mathrm{~d}, 1 \mathrm{H}, J=2.0), 7.09(\mathrm{dd}, 1 \mathrm{H}, J=2.0, J=8.7), 7.39-7.85(\mathrm{~m}, 5 \mathrm{H}), 7.75(\mathrm{~m}, 2 \mathrm{H}), 7.85(\mathrm{~m}$, $1 \mathrm{H}), 8.01(\mathrm{~s}, 1 \mathrm{H}), 8.60(\mathrm{~s}, 1 \mathrm{H}), 8.86(\mathrm{~d}, 1 \mathrm{H}, J=8.7), 9.46(\mathrm{~s}, 1 \mathrm{H}) ;{ }^{13} \mathrm{C} \mathrm{NMR}\left(\mathrm{CDCl}_{3}\right): 23.4\left(\mathrm{CH}_{3}\right), 44.9$ $\left(\mathrm{CH}_{2}\right), 46.2\left(2 \mathrm{CH}_{3}\right), 57.5\left(\mathrm{CH}_{2}\right), 70.8\left(\mathrm{CH}_{2}\right), 92.8(\mathrm{CH}), 109.8(\mathrm{Cq}), 110.5(\mathrm{Cq}), 114.3(\mathrm{CH}), 117.6$ (Cq), $119.5(\mathrm{Cq}), 119.9(\mathrm{CH}), 123.1(\mathrm{Cq}), 123.9(\mathrm{CH}), 124.6(\mathrm{CH}), 125.3(\mathrm{CH}), 125.5(\mathrm{CH}), 126.5$ $(2 \mathrm{CH}), 127.0(\mathrm{CH}), 127.3(\mathrm{CH}), 127.5(\mathrm{CH}), 125.7(2 \mathrm{CH}+\mathrm{Cq}), 129.9(\mathrm{Cq}), 130.4 \mathrm{Cq}), 135.9(\mathrm{Cq})$, $136.7(\mathrm{Cq}), 140.6(\mathrm{Cq}), 157.2(\mathrm{Cq}), 167.8(\mathrm{CO}), 168.7(\mathrm{CO})$; MS (IS) $528(\mathrm{M}+1)^{+}$.

\section{2-\{[(tert-Butyldimethyl)silyl]oxy\}-6-methylnaphtho[2,3-a]pyrrolo[3,4-c]carbazole-5,7(6H,14H)-}

dione (46). $\mathrm{Mp}: 172{ }^{\circ} \mathrm{C}$; Rf (petroleum ether EtOAc 3/7) : 0.36 ; IR (KBr): v 3319, 2953, 2928, 2856, 1752, 1682, 1378, $894 ;{ }^{1} \mathrm{H}$ NMR (DMSO-d $): 0.28(\mathrm{~s}, 6 \mathrm{H}), 1.02(\mathrm{~s}, 9 \mathrm{H}), 3.00(\mathrm{~s}, 3 \mathrm{H}), 6.87(\mathrm{dd}, 1 \mathrm{H}, J=$ $1.9, J=10.6), 7.08(\mathrm{~d}, 1 \mathrm{H}, J=1.9), 7.57(\mathrm{~m}, 2 \mathrm{H}), 8.00-8.07(\mathrm{~m}, 2 \mathrm{H}), 8.59(\mathrm{~d}, 1 \mathrm{H}, J=8.7), 8.97(\mathrm{~s}, 1 \mathrm{H})$, $9.32(\mathrm{~s}, 1 \mathrm{H}), 12.82\left(\mathrm{~s}, 1 \mathrm{H}\right.$, exchangeable $\left.\mathrm{D}_{2} \mathrm{O}\right) ;{ }^{13} \mathrm{C}$ NMR $\left(\mathrm{DMSO}-d_{6}\right):-3.5\left(2 \mathrm{CH}_{3}\right), 24.2\left(\mathrm{CH}_{3}\right), 25.5$ $\left(3 \mathrm{CH}_{3}\right), 71.8(\mathrm{Cq}), 102.8(\mathrm{CH}), 110.6(\mathrm{Cq}), 115.7(\mathrm{CH}), 118.5(\mathrm{Cq}), 121.7(\mathrm{CH}), 124.0(\mathrm{Cq}), 124.7$ $(\mathrm{CH}), 125.2(\mathrm{CH}), 127.3(\mathrm{CH}), 127.7(\mathrm{CH}), 128.9(\mathrm{CH}), 129.3(\mathrm{Cq}), 129.5(\mathrm{CH}), 132.1(\mathrm{Cq}), 132.5$ (Cq), $136.5(\mathrm{Cq}), 140.7(\mathrm{Cq}), 141.4(\mathrm{Cq}), 145.3(\mathrm{Cq}), 154.9(\mathrm{Cq}), 169.9(\mathrm{CO}), 170.8(\mathrm{CO})$; MS (IS) : $481(\mathrm{M}+1)^{+}$.

\section{2-[2-(Dimethylamino)ethyl]-6-methylnaphtho[2,3-a]pyrrolo[3,4-c] carbazole-5,7(6H,14H)-dione}

(47). Mp : $168{ }^{\circ} \mathrm{C}$; Rf (acetone) : $0.18 ; \operatorname{IR}(\mathrm{KBr}): v 3427,2943,1750,1695,1380,1174,726 ;{ }^{1} \mathrm{H}$ NMR (DMSO- $\left.d_{6}\right): 2.48(\mathrm{~s}, 6 \mathrm{H}), 2.71(\mathrm{t}, 2 \mathrm{H}, J=5.0), 3.07(\mathrm{~s}, 3 \mathrm{H}), 4.16(\mathrm{~d}, 2 \mathrm{H}, J=5), 6.91(\mathrm{dd}, 1 \mathrm{H}, J=$ $2, J=8.5), 7.08(\mathrm{~s}, 1 \mathrm{H}), 7.51(\mathrm{~m}, 2 \mathrm{H}), 8.02(\mathrm{~d}, 1 \mathrm{H}, J=8.5), 8.10(\mathrm{~d}, 1 \mathrm{H}, J=8.5), 8.59(\mathrm{~d}, 1 \mathrm{H}, J=8.5)$, $9.01(\mathrm{~s}, 1 \mathrm{H}), 8.35(\mathrm{~s}, 1 \mathrm{H}), 12.83\left(\mathrm{~s}, 1 \mathrm{H}\right.$, exchangeable $\left.\mathrm{D}_{2} \mathrm{O}\right) ;{ }^{13} \mathrm{C}$ NMR $\left(\right.$ DMSO- $\left.d_{6}\right): 23.9\left(\mathrm{CH}_{3}\right), 46.5$ $\left(2 \mathrm{CH}_{3}\right), 58.7\left(\mathrm{CH}_{2}\right), 66.8\left(\mathrm{CH}_{2}\right), 97.9(\mathrm{CH}), 112.0(\mathrm{CH}), 112.9(\mathrm{Cq}), 113.7(\mathrm{Cq}), 117.8(\mathrm{Cq}), 119.7(\mathrm{Cq})$, $123.0(\mathrm{Cq}+\mathrm{CH}), 125.4(\mathrm{Cq}+\mathrm{CH}), 126.1(\mathrm{Cq}), 126.6(\mathrm{Cq}), 128.6(\mathrm{CH}), 128.9(\mathrm{CH}), 130.4(\mathrm{CH}), 131.0^{\mathrm{S} 15}$ 
(CH), $133.6(\mathrm{CH}), 133.8(\mathrm{Cq}), 141.9(\mathrm{Cq}), 142.9(\mathrm{Cq}), 160.0(\mathrm{Cq}), 171.3(\mathrm{CO}), 172.2(\mathrm{CO})$; MS (IS) : $438(\mathrm{M}+1)^{+}$. 
Elemental analysis Table.

\begin{tabular}{|c|c|c|c|}
\hline Compound \# & Anal. & Calculated, $\mathrm{C}, \mathrm{H}, \mathrm{N}$ & Found, C, H, N \\
\hline 3 & $\mathrm{C}_{19} \mathrm{H}_{13} \mathrm{BrN}_{2} \mathrm{O}_{4} \mathrm{~S}$ & $51.25,2.94,6.29$ & $51.43,3.06,6.17$ \\
\hline 4 & $\mathrm{C}_{14} \mathrm{H}_{18} \mathrm{OSn}$ & $52.39,5.65$. & $52.63,5.49$. \\
\hline 8 & $\mathrm{C}_{24} \mathrm{H}_{18} \mathrm{~N}_{2} \mathrm{O}_{3}$ & $75.38,4.74,7.33$ & $75.70,4.62,7.21$ \\
\hline 10 & $\mathrm{C}_{30} \mathrm{H}_{22} \mathrm{~N}_{2} \mathrm{O}_{5} \mathrm{~S}$ & $68.95,4.24,5.36$ & $68.64,4.41,5.23$ \\
\hline 11 & $\mathrm{C}_{23} \mathrm{H}_{16} \mathrm{~N}_{2} \mathrm{O}_{3}$ & $74.99,4.38,7.60$. & $75.31,4.50,7.55$ \\
\hline 12 & $\mathrm{C}_{29} \mathrm{H}_{20} \mathrm{~N}_{2} \mathrm{O}_{5} \mathrm{~S}$ & $68.49,3.96,5.51$. & $68.73,4.03,5.37$. \\
\hline 13 & $\mathrm{C}_{24} \mathrm{H}_{15} \mathrm{~F}_{3} \mathrm{~N}_{2} \mathrm{O}_{5} \mathrm{~S}$ & $57.60,3.02,5.60$. & $57.89,3.12,5.49$. \\
\hline 14 & $\mathrm{C}_{30} \mathrm{H}_{19} \mathrm{~F}_{3} \mathrm{~N}_{2} \mathrm{O}_{7} \mathrm{~S}_{2}$ & $56.25,2.99,4.37$ & $56.03,3.14,4.48$ \\
\hline 15 & $\mathrm{C}_{23} \mathrm{H}_{14} \mathrm{~N}_{2} \mathrm{O}_{2}$ & $78.84,4.03,8.00$. & $79.13,4.19,8.08$. \\
\hline 16 & $\mathrm{C}_{29} \mathrm{H}_{18} \mathrm{~N}_{2} \mathrm{O}_{4} \mathrm{~S}$ & $71.01,3.70,5.71$ & $70.76,3.84,5.87$ \\
\hline 17 & $\mathrm{C}_{26} \mathrm{H}_{19} \mathrm{BrN}_{2} \mathrm{O}_{5} \mathrm{~S}$ & $56.63,3.47,5.08$ & $56.32,3.59,5.24$ \\
\hline 18 & $\mathrm{C}_{26} \mathrm{H}_{19} \mathrm{BrN}_{2} \mathrm{O}_{5} \mathrm{~S}$ & $56.63,3.47,5.08$ & $56.87,3.60,5.01$. \\
\hline 21 & $\mathrm{C}_{36} \mathrm{H}_{26} \mathrm{~N}_{2} \mathrm{O}_{6} \mathrm{~S}$ & $70.35,4.26,4.56$ & $70.63,4.40,4.67$. \\
\hline 22 & $\mathrm{C}_{36} \mathrm{H}_{26} \mathrm{~N}_{2} \mathrm{O}_{6} \mathrm{~S}$ & $70.35,4.26,4.56$ & $70.69,4.05,4.63$ \\
\hline 23 & $\mathrm{C}_{28} \mathrm{H}_{19} \mathrm{~N}_{3} \mathrm{O}_{5} \mathrm{~S}$ & $66.00,3.76,8.25$ & $65.74,3.91,8.28$ \\
\hline 24 & $\mathrm{C}_{37} \mathrm{H}_{25} \mathrm{~F}_{3} \mathrm{~N}_{2} \mathrm{O}_{8} \mathrm{~S}_{2}$ & $59.51,3.37,3.75$ & $59.23,3.52,3.58$ \\
\hline 25 & $\mathrm{C}_{37} \mathrm{H}_{25} \mathrm{~F}_{3} \mathrm{~N}_{2} \mathrm{O}_{8} \mathrm{~S}_{2}$ & $59.51,3.37,3.75$ & $59.28,3.50,3.64$ \\
\hline 26 & $\mathrm{C}_{29} \mathrm{H}_{18} \mathrm{~F}_{3} \mathrm{~N}_{3} \mathrm{O}_{7} \mathrm{~S}_{2}$ & $54.29,2.83,6.55$ & $54.03,2.72,6.67$ \\
\hline 27 & $\mathrm{C}_{30} \mathrm{H}_{20} \mathrm{~N}_{2} \mathrm{O}_{3}$ & $78.93,4.42,6.14$ & $79.14,4.30,6.29$ \\
\hline 28 & $\mathrm{C}_{30} \mathrm{H}_{20} \mathrm{~N}_{2} \mathrm{O}_{3}$ & $78.93,4.42,6.14$. & $79.19,4.35,6.22$ \\
\hline 29 & $\mathrm{C}_{23} \mathrm{H}_{14} \mathrm{~N}_{2} \mathrm{O}_{3}$ & $75.40,3.85,7.65$ & $75.76,3.76,7.74$ \\
\hline 30 & $\mathrm{C}_{23} \mathrm{H}_{14} \mathrm{~N}_{2} \mathrm{O}_{3}$ & $75.40,3.85,7.65$ & $75.63,3.70,7.54$ \\
\hline 31 & $\mathrm{C}_{29} \mathrm{H}_{25} \mathrm{~N}_{3} \mathrm{O}_{3}$ & $75.14,5.44,9.07$ & $75.01,5.32,8.95$ \\
\hline 32 & $\mathrm{C}_{27} \mathrm{H}_{23} \mathrm{~N}_{3} \mathrm{O}_{2}$ & $76.94,5.50,9.97$ & $77.26,5.67,10.08$. \\
\hline 33 & $\mathrm{C}_{26} \mathrm{H}_{18} \mathrm{~N}_{2} \mathrm{O}_{2}$ & $79.98,4.65,7.17$ & $79.69,4.73,7.23$ \\
\hline 34 & $\mathrm{C}_{26} \mathrm{H}_{20} \mathrm{~N}_{2} \mathrm{O}_{4}$ & $73.57,4.75,6.60$ & $73.35,4.71,6.52$ \\
\hline
\end{tabular}




\begin{tabular}{|l|l|l|l|}
\hline $\mathbf{3 5}$ & $\mathrm{C}_{22} \mathrm{H}_{11} \mathrm{NO}_{3}$ & $78.33,3.29,4.15$. & $78.09,3.48,4.06$. \\
\hline $\mathbf{3 6}$ & $\mathrm{C}_{26} \mathrm{H}_{21} \mathrm{~N}_{3} \mathrm{O}_{2}$ & $76.64,5.19,10.31$. & $76.76,5.27,10.16$. \\
\hline $\mathbf{3 7}$ & $\mathrm{C}_{26} \mathrm{H}_{21} \mathrm{~N}_{3} \mathrm{O}_{3}$ & $73.74,5.00,9.92$. & $74.02,4.9 .5,10.11$. \\
\hline $\mathbf{3 8}$ & $\mathrm{C}_{26} \mathrm{H}_{21} \mathrm{~N}_{3} \mathrm{O}_{3}$ & $73.74,5.00,9.92$. & $74.53,5.09,10.04$. \\
\hline $\mathbf{3 9}$ & $\mathrm{C}_{27} \mathrm{H}_{23} \mathrm{~N}_{3} \mathrm{O}_{3}$ & $74.13,5.30,9.60$. & $73.88,5.18,9.58$. \\
\hline $\mathbf{4 0}$ & $\mathrm{C}_{27} \mathrm{H}_{23} \mathrm{~N}_{3} \mathrm{O}_{3}$ & $74.13,5.30,9.60$. & $74.32,5.45,9.43$. \\
\hline $\mathbf{4 2}$ & $\mathrm{C}_{26} \mathrm{H}_{21} \mathrm{~N}_{3} \mathrm{O}_{4}$ & $71.06,4.82,9.56$. & $70.87,4.99,9.45$. \\
\hline $\mathbf{4 3}$ & $\mathrm{C}_{26} \mathrm{H}_{21} \mathrm{~N}_{3} \mathrm{O}_{4}$ & $71.06,4.82,9.56$. & $71.27,4.65,9.48$. \\
\hline $\mathbf{4 4}$ & $\mathrm{C}_{25} \mathrm{H}_{18} \mathrm{~N}_{2} \mathrm{O}_{5}$ & $70.42,4.25,6.57$. & $70.25,4.43,6.68$. \\
\hline $\mathbf{4 5}$ & $\mathrm{C}_{25} \mathrm{H}_{18} \mathrm{~N}_{2} \mathrm{O}_{5}$ & $70.42,4.25,6.57$. & $70.33,4.32,6.34$. \\
\hline $\mathbf{4 6}$ & $\mathrm{C}_{34} \mathrm{H}_{29} \mathrm{~N}_{3} \mathrm{O}_{3}$ & $77.40,5.54,7.96$. & $77.11,5.67,8.09$. \\
\hline $\mathbf{4 7}$ & $\mathrm{C}_{29} \mathrm{H}_{28} \mathrm{~N}_{2} \mathrm{O}_{3} \mathrm{Si}$ & $72.47,5.87,5.83$. & $72.74,5.70,5.95$. \\
\hline
\end{tabular}

